\title{
Introduction to Logical Information Systems
}

\author{
S. Ferré ${ }^{\mathrm{a}, 1}$, O. Ridoux ${ }^{\mathrm{b}}$ \\ ${ }^{a} U W A$, Penglais, Aberystwyth SY23 3DB, UK, \\ Tel: +44 1970 621922, Fax: +441970 622455, \\ Email:sbf@aber.ac.uk \\ ${ }^{\mathrm{b}}$ Irisa/IFSIC, Campus de Beaulieu, 35042 Rennes cedex, France, \\ Tel: +332 998473 30, Fax: +332998471 71, \\ Email: ridoux@irisa.fr
}

\begin{abstract}
Logical Information Systems (LIS) use logic in a uniform way to describe their contents, to query it, to navigate through it, to analyze it, and to maintain it. They can be given an abstract specification that does not depend on the choice of a particular logic, and concrete instances can be obtained by instantiating this specification with a particular logic. In fact, a logic plays in a LIS the role of a schema in data-bases. We present the principles of logical information systems, the constraints they impose on the expression of logics, and hints for their effective implementation.
\end{abstract}

Key words: information systems, information search and retrieval, query formulation, representation languages, deduction and theorem proving.

\section{Introduction}

Several researchers have recognized the name problem in information systems (Gifford, Jouvelot, Sheldon, and O'Toole, 1991; Gopal and Manber, 1999). In these systems, things are given names and very often a thing has only a few names (frequently only one). For instance, in the most rudimentary information systems, like hierarchical file systems, a thing is a file and its name is the only path that leads from the root to the file.

1 This work was achieved at Irisa (Rennes, France), and funded by a scholarship from CNRS and Région Bretagne.

Preprint submitted to Elsevier Science 13th January 2003 
The problem with having only a few names is that they must be very carefully chosen to tackle for all future usages of the things. Experience shows that this is impossible to do for a wide range of future usages. For instance, there is no hierarchical organization of many pieces of software that fits all the needs of software development: programming, testing, documenting, debugging, etc. A non-IT example is a cook-book. Cook-books are often organized following the course of a meal, and it is thus very difficult to search for a recipe according to other criteria, like the (un)availability of a oven, or a diet constraint. Note that even electronic cook-books often follow this structure. Electronic or not, the classical solution to this problem is to search in the whole information system/document to find the desired thing. However, this is only a partial solution because query language used in search engines are often too restricted.

There have been several attempts for solving the name problem. We mention here only two of them that we have selected because they illustrate the difficulty of the enterprise, SFS (Gifford, Jouvelot, Sheldon, and O'Toole, 1991) and HAC (Gopal and Manber, 1999). They have in common to combine hierarchical naming and boolean querying.

Hierarchical naming is frequently found in computer tools: e.g., file systems, book-marks, or menus. In this model, searching is done by navigating in a classification structure that is often built and maintained manually. Navigating implies a notion of place; being in a place, and going to another place. A notion of neighborhood helps specifying the "other places" relatively to the place one is currently in. Many applications require that a place is a place to read from as well as a place to write on.

Boolean querying is often found in information servers such as search engines on the Web (e.g., Google). In this model, searching is done by using queries, generally expressed in a kind of propositional logic. A well-recognized difficulty of this model is the necessity of having a good knowledge of the terminology used in the information system, and of having a precise idea of what is searched for. However, it is easier to recognize an object than to describe it, and the necessity of expressing a query can repel casual users.

Then, which search model should be preferred: navigation or querying? In fact, it depends on situations, and it is sometimes needed to use both of them in the same search. For instance, someone could wish to begin his search by a query and then refine it with navigation. Hybrid systems combining hierarchical classification and boolean querying have been proposed in the domain of file systems:

— SFS (Semantic File System, Gifford, Jouvelot, Sheldon, and O'Toole, 1991) extends the hierarchical model of usual file systems with virtual directories that correspond to queries. These queries concern file properties that are automat- 
ically extracted by transducers, and are expressed with valued attributes. So, two organization and storage methods coexist: the standard hierarchy that gives a name to files, and virtual directories that enable associative searches on intrinsic file properties. Unfortunately, these two methods cannot be com-

bined freely in general. In particular, virtual directories are not places to write into.

- HAC (Hierarchy And Content, Gopal and Manber, 1999) also uses queries to build directories based on file contents, but these directories are integrated in the hierarchy. This enables to combine hierarchy and contents in searches. However, users are allowed to move a file in a directory even if it does not satisfy the query associated to the directory, which results in consistency problems.

The drawback of these hybrid systems is their lack of consistency. Indeed, they have two search models that are not tightly connected, which makes it difficult to switch from one model to the other, and to combine both in the same search.

We propose a scheme called Logical Information Systems (LIS) in which queries are really places to read from and to write into. The scheme is flexible in the sense that the neighborhood relation is very dense (i.e., things have many names). It incurs no inconsistency or dangling links problem, because the neighborhood relation is managed automatically. Finally, it supports both querying and navigation, and arbitrary combinations of both because names and queries belong to the same language (Godin, Missaoui, and April, 1993; Lindig, 1995). This scheme is based on a variant of Formal Concept Analysis (Barbut and Monjardet, 1970; Wille, 1982; Ganter and Wille, 1999) called Logical Concept Analysis (Ferré and Ridoux, 2000).

The article is organized as follows. Section 2 presents the principles of Logical Concept Analysis, and then Section 3 shows how it can be used for navigating and querying. How to create and update the content of a LIS is presented in Section 4. These sections refer to a logic passed as a parameter which is to be used for naming, querying and navigating. Section 5 presents other functions of a LIS like automated updating, data-mining and learning. Section 6 explains how all this can be done practically. Finally, conclusions and perspectives are given in Section 7.

\section{Logical Concept Analysis}

The origin of this work is the search for flexible organizations for managing, updating, querying, or navigating in data. In this context, several roles are 
played by possibly different people: e.g., designer, administrator, and enduser. Hierarchical organizations are not flexible, and updating, querying and navigation are difficult to conciliate (see for instance the view update problem in data-bases (Keller, 1985)). The literature shows that Formal Concept Analysis (FCA, Ganter and Wille, 1999) is a good candidate for supporting querying and navigation.

The basis of FCA is a formal context that associates attributes to objects; objects are the things of the introduction of this article, and the collection of attributes associated to one thing/object is its name. FCA has received attention for its application in many domains such as in software engineering (Snelting, 1998; Lindig, 1995; Krone and Snelting, 1994). The interest of FCA as a navigation tool in general has also been recognized (Godin, Missaoui, and April, 1993; Lindig, 1995; Vogt and Wille, 1994). However, we feel it is not flexible enough as far as the naming of things is concerned, and the literature on FCA insists more on analyzing a given context than on managing evolving contexts. In this section, we present an extension to FCA that allows for a richer name language.

The variety of application domains brings the need for more sophisticated formal contexts than the mere presence/absence of attributes. For instance, many application domains use numerical values (e.g., lengths, prices, ages), and the need to express negation and disjunction is often felt. In a much more specialized scope, it is imaginable to use the type of software components as search keys (Di Cosmo, 1995). So, one cannot fix a priori limits to the sophistication of attributes. Several enrichments to the attribute structure have been proposed: e.g., many-valued attributes (Ganter and Wille, 1999), and first-order terms (Chaudron and Maille, 1998). However, not a single extended FCA framework covers all the concrete domains, and no one can pretend covering all the concrete domains to come.

For the same reasons, logic has already been proposed, in information retrieval, for expressing object descriptions and queries (van Rijsbergen, 1986; Meghini, Sebastiani, Straccia, and Thanos, 1993). In this setting, the relevance of an object to a query relies on logical inference. As with Description Logics (Brachman, 1979; Napoli, 1997), we use an exact relevance relation, called subsumption, as some applications may need precise retrieval mechanisms; but uncertain relevance has also been considered (Crestani and Lalmas, 2001). See also a discussion on the management of uncertainty in the conclusion of this article. So, we propose to construct a more general framework for concept analysis, Logical Concept Analysis (LCA), in which attributes are replaced by the formulas of a logic . Moreover, we make this logic a parameter of LCA. This allows for instantiating the general framework by merely filling in a dedicated logic. 


\subsection{Logic}

Logical Concept Analysis (LCA) is a generalization of FCA, where sets of attributes are replaced by formulas of an (almost) arbitrary logic. More details about the relation with previous works in FCA can be found in (Ferré and Ridoux, 2000), and all proofs can be found in (Ferré and Ridoux, 1999).

We first define what we call a logic in this article.

Definition 1 (logic) A logic is a 6-tuple $(\mathcal{L}, \sqsubseteq, \sqcap, \sqcup, \top, \perp)$, where

- $\mathcal{L}$ is the language of formulas,

- $\sqsubseteq$ is the subsumption relation (pre-order over $\mathcal{L}$ ),

- $\sqcap$ and $\sqcup$ are respectively conjunction and disjunction (binary operations),

- $\top$ and $\perp$ are respectively tautology and contradiction (constant formulas).

Such a logic must form a lattice (Davey and Priestley, 1990), whose order is derived in the usual way from the pre-order $\sqsubseteq$, and such that $\sqcap$ and $\sqcup$ are respectively the infimum (greatest lower bound) and the supremum (least upper bound), and $\top$ and $\perp$ are respectively the top and the bottom. The notation $\mathcal{L}$ can be used as a name for the logic lattice.

If $f \sqsubseteq g$ and $g \sqsubseteq f, f$ and $g$ are called logically equivalent, which is denoted as $f \equiv g$; we consider them as different representations of the same equivalence class, and in fact we will consider that elements of $\mathcal{L}$ are the equivalence classes. We just assume, for practical reasons, that operations $\sqsubseteq$, $\sqcap$, and $\sqcup$ are computable. Some semantics is usually used to define a logic, but we delay the discussion about it until Section 6.2 as we do not need it here. Just keep in mind that formulas are here interpreted by sets of individuals or objects (like in Description Logics), rather than by truth values (like in first-order logic). In order to clarify things, here is an example of a logic.

Example 2 (Propositional logic) An example of logic that can be used in $L C A$ is propositional logic. On the syntactic side, the set of propositions $\mathcal{P}$ contains atomic propositions (taken in a set $\mathcal{A}$ ), formulas 0 and 1 , and is closed under binary connectives $\wedge$ and $\vee$, and unary connective $\neg$. We say that a proposition $p$ is subsumed by another one $q$ if $\neg p \vee q$ is a valid proposition $(p \vDash q)$. Then, $(\mathcal{P}, \vDash, \wedge, \vee, 1,0)$ satisfies Definition 1 , because it is the wellknown boolean algebra.

This example shows that though the interface of the logic is limited to the tuple $(\mathcal{L}, \sqsubseteq, \sqcap, \sqcup, \top, \perp)$, an actual logic may have more connectives: $\neg$ in this example.

We now define the main notions and results of LCA: context, concept lattice, 
and contextualized subsumption.

\subsection{Logical context and Galois connection}

A logical context plays the role of tables in a database, as it gathers the knowledge one has about objects of interest (e.g., files, bibliographic references, recipes).

Definition 3 (logical context) A logical context (context for short) is a triple $K=(\mathcal{O}, \mathcal{L}, d)$ where:

- $\mathcal{O}$ is a finite set of objects,

- $\mathcal{L}$ is a logic (as in Definition 1),

- $d$ is a mapping from $\mathcal{O}$ to $\mathcal{L}$ that describes each object by a formula.

Then, we define two mappings between sets of objects $\left(2^{\mathcal{O}}\right)$ and formulas $(\mathcal{L})$ in a context $K$, that we prove to be a Galois connection (Davey and Priestley, 1990). A first mapping $\tau_{K}$ connects each formula $f$ to its instances, i.e., objects whose description is subsumed by $f ; \tau_{K}(f)$ is the extent of $f$. A second mapping $\sigma_{K}$ connects each set of objects $O \subseteq \mathcal{O}$ to the most precise formula subsuming all descriptions of objects in $O ; \sigma_{K}(O)$ is the intent of $O$.

Definition 4 (mappings $\tau_{K}$ and $\left.\sigma_{K}\right)$ Let $K=(\mathcal{O}, \mathcal{L}, d)$ be a context, $O \subseteq \mathcal{O}$, and $f \in \mathcal{L}$

- $\sigma_{K}(\sigma$ for short $): 2^{\mathcal{O}} \rightarrow \mathcal{L}, \sigma_{K}(O):=\bigsqcup_{o \in O} d(o)$

- $\tau_{K}(\tau$ for short $): \mathcal{L} \rightarrow 2^{\mathcal{O}}, \tau_{K}(f):=\{o \in \mathcal{O} \mid d(o) \sqsubseteq f\}$

Lemma 5 (Galois connection) Let $K$ be a context. The pair $\left(\sigma_{K}, \tau_{K}\right)$ is a Galois connection because

$\forall O \subseteq \mathcal{O}, f \in \mathcal{L}: \sigma_{K}(O) \sqsubseteq f \Longleftrightarrow O \subseteq \tau_{K}(f)$

In the following, we will drop the subscript $K$ when possible.

Example 6 (Triv) An example context will illustrate the rest of our development on LCA. Context $K_{\text {Triv }}$ is deliberately small and simple as it is aimed at illustrating theoretical notions, and not at showing a realistic application of LCA. The logic used in this context is propositional logic $\mathcal{P}$ (see Example 2) with a set of atomic propositions $\mathcal{A}=\{a, b, c\}$. We define context $K_{\text {Triv }}$ by $\left(\mathcal{O}_{\text {Triv }}, \mathcal{P}, d_{\text {Triv }}\right)$, where $\mathcal{O}_{\text {Triv }}=\{x, y, z\}$, and where $d_{\text {Triv }}=\{x \mapsto a, y \mapsto b, z \mapsto c \wedge(a \vee b)\}$.

A Logical Information System (LIS) is essentially a logical context equipped with navigation and management tools. Formulas serve as queries, and extents 
as answers via mapping $\tau_{K}$. This is only a rough description. We will see in Section 3 that a LIS answer can also be a formula. For illustration purpose, we consider a bibliographical information system.

Example $7(B i b)$ Let $B i b=\left(\mathcal{O}, \mathcal{P}_{v}, d\right)$ be a logical context where objects are bibliographical references, whose description are composed of a type (e.g., article, in-proceedings), a list of authors, a title, and a year of publication. The logic $\mathcal{P}_{v}$, used for expressing descriptions, is similar to the propositional logic $\mathcal{P}$ (see Example 2) except that atoms are replaced by valued attributes in the form attr value: attr is the name of an attribute (e.g., author, year), and value expresses a logical property about the value of the attribute. For instance, numerical attributes can be described in an interval logic (e.g., year in 1990..2000, year in 1995), and string attributes can be described by a string (e.g., title is "Logical Information Systems") or a substring (e.g., title contains "System"). We now show as an example the logical description of our article on LCA (Ferré and Ridoux, 2000).

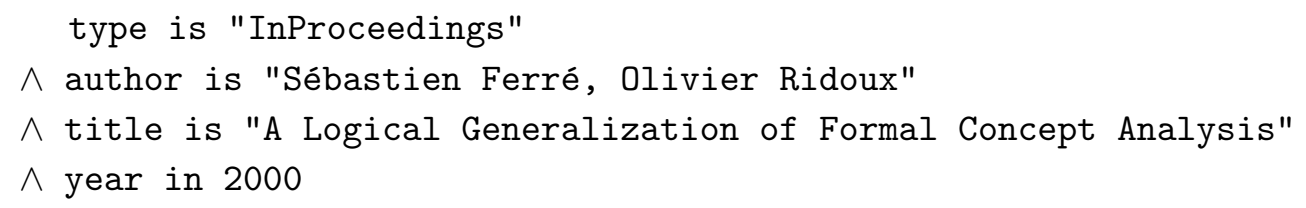

In the sequel of this article, the context Bib refers to all ICCS publications until the year 1999, which consists in 209 objects.

\subsection{Concept lattice and labelling}

A formal concept, central notion of LCA, is the association of a set of objects and of a formula, which is stable for the Galois connection $(\sigma, \tau)$.

Definition 8 (formal concept) In a context $K=(\mathcal{O}, \mathcal{L}, d)$, a formal concept (concept for short) is a pair $c=(O, f)$ where $O \subseteq \mathcal{O}$, and $f \in \mathcal{L}$, such that $\sigma_{K}(O) \equiv f$ and $\tau_{K}(f)=O$.

The set of objects $O$ is the concept extent (written ext $(c)$ ), whereas formula $f$ is its intent (written int $(c)$ ).

We write $={ }^{c}$ for concept equality. The set of all concepts that can be built in a context $K$ is denoted by $\mathcal{C}_{K}$, and is partially ordered by $\leq^{c}$ defined below. The fundamental theorem of LCA is that $\left\langle\mathcal{C}_{K} ; \leq^{c}\right\rangle$ forms a lattice, which is finite, hence complete.

Definition 9 (partial order $\leq^{c}$ ) Let $c_{1}$ and $c_{2}$ be in $\mathcal{C}_{K}$, 
$c_{1} \leq^{c} c_{2} \Longleftrightarrow \operatorname{ext}\left(c_{1}\right) \subseteq \operatorname{ext}\left(c_{2}\right)$

(could be defined equivalently by $\left.\operatorname{int}\left(c_{1}\right) \sqsubseteq \operatorname{int}\left(c_{2}\right)\right)$.

Theorem 10 (concept lattice) Let $K$ be a context. The partially ordered set $\left\langle\mathcal{C}_{K} ; \leq^{c}\right\rangle$ is a finite lattice, whose supremum and infimum are as follows for every set of indices $J$ :

- $\bigvee_{j \in J}^{c}\left(O_{j}, f_{j}\right)={ }^{c}\left(\tau_{K}\left(\sigma_{K}\left(\bigcup_{j \in J} O_{j}\right)\right), \sqcup_{j \in J} f_{j}\right)$

- $\bigwedge_{j \in J}^{c}\left(O_{j}, f_{j}\right)={ }^{c}\left(\bigcap_{j \in J} O_{j}, \sigma_{K}\left(\tau_{K}\left(\sqcap_{j \in J} f_{j}\right)\right)\right)$

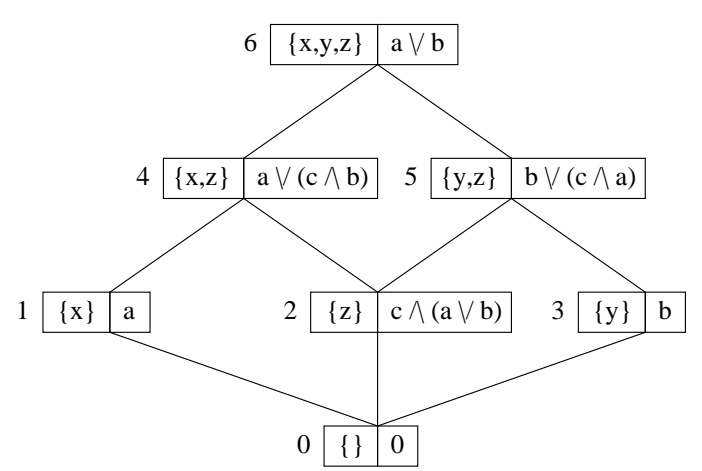

\begin{tabular}{ll|l|}
\cline { 2 - 3 } concept & extent & intent \\
\hline
\end{tabular}

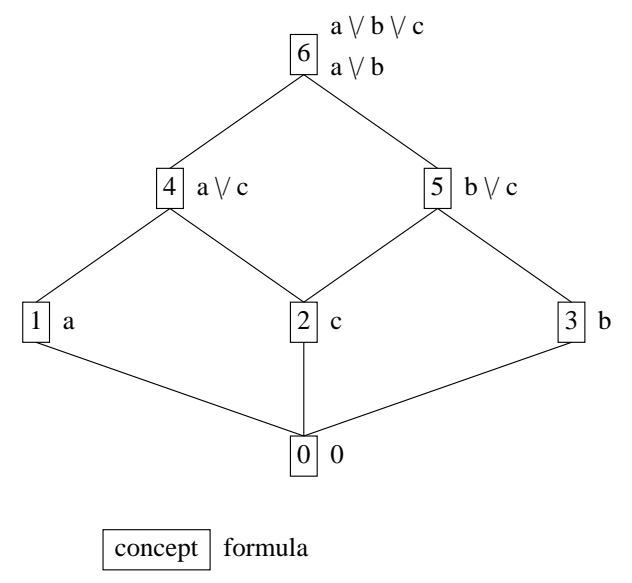

(b)

Figure 1. The concept lattice of context $K_{\text {Triv }}$ (a) and its labelling (b).

Example 11 (Triv) Figure 1.(a) represents the Hasse diagram of the concept lattice of context $K_{\text {Triv }}$ (introduced in Example 6). Concepts are represented by a number and a box containing their extent on the left, and their intent on the right. The higher concepts are placed in the diagram the greater they are for partial order $\leq^{c}$. It can be observed that the concept lattice is not isomorphic to the power-set lattice of objects $\left\langle 2^{\mathcal{O}} ; \subseteq\right\rangle$. Indeed, set $\{x, y\}$ is not the extent of any concept, because $\tau(\sigma(\{x, y\}))=\tau(a \vee b)=\{x, y, z\}$.

To make the concept lattice more readable, it is possible to label it with formulas and objects. Mapping $\mu$ labels with a formula $f$ the concept whose extent is the extent of $f$. Mapping $\gamma$ labels with an object $o$ the concept whose intent is the intent of $o$, that is its description.

Definition 12 (labelling) Let $K=(\mathcal{O}, \mathcal{L}, d)$ be a context, o $\in \mathcal{O}$, and $f \in \mathcal{L}$,

- $\mu_{K}(\mu$ for short $): \mathcal{L} \rightarrow \mathcal{C}_{K}, \operatorname{ext}\left(\mu_{K}(f)\right)=\tau_{K}(f)$

- $\gamma_{K}(\gamma$ for short $): \mathcal{O} \rightarrow \mathcal{C}_{K}$, int $\left(\gamma_{K}(o)\right) \equiv d(o)$.

The interesting thing with this labelling is that it enables to retrieve all data of the context: an object $o$ satisfies $(\sqsubseteq)$ a formula $f$ in some context $K$ if and 
only if the concept labelled with $o$ is below $\left(\leq^{c}\right)$ the concept labelled with $f$ in the concept lattice of $K$.

Lemma 13 (labelling) Under the conditions of Definition 12,

$$
d(o) \sqsubseteq f \Longleftrightarrow \gamma_{K}(o) \leq^{c} \mu_{K}(f) \text {. }
$$

Example 14 (Triv) Figure 1.(b) represents the same concept lattice as Figure 1.(a) (see also Example 11), but its concepts are decorated with the $\mu$ labelling instead of with the extents and intents. Formulas of the form $\vee A$ where $A \subseteq \mathcal{A}(\vee \emptyset \equiv 0)$ are placed on the right of the concept that they label. For instance, concept 1 is labelled by formula a (i.e., $\mu(a)={ }^{c} 1$ ). In Figure 1.(b) we have restricted labels to be formulas of the form $\vee A$, but it is only to have a finite number of labels that are not all in the formal context.

\subsection{Contextualized subsumption}

In most contexts, it is possible to order some properties, although they are not comparable by $\sqsubseteq$. For instance, if in some context every bird flies, then we can say that property "bird" is contextually subsumed by the property "fly", although we have not necessarily bird $\sqsubseteq f l y$ in $\mathcal{L}$. We introduce a contextualized subsumption as a generalization of implications between attributes that are used in standard CA for knowledge acquisition processes (Ganter and Wille, 1999; Snelting, 1998).

Definition 15 (contextualized subsumption) Let $K=(\mathcal{O}, \mathcal{L}, d)$ be $a$ context, and $f, g \in \mathcal{L}$. One says that $f$ is contextually subsumed by $g$ in context $K$, which is noted $f \sqsubseteq_{K} g$, if and only if $\tau_{K}(f) \subseteq \tau_{K}(g)$, i.e., if every object that satisfies $f$ also satisfies $g$.

Every arc of $\sqsubseteq_{K}$ is called a contextualized implication.

Contextualized subsumption has a close connection with the concept lattice.

\section{Theorem 16 (contextualized subsumption vs. concept lattice)}

Let $K$ be a context. The partially ordered set of formulas $\left\langle\mathcal{L} ; \Xi_{K}\right\rangle$, derived in the usual way from the pre-order $\sqsubseteq_{K}$, is isomorphic to the concept lattice $\left\langle\mathcal{C}_{K} ; \leq^{c}\right\rangle$. The morphism from formulas to concepts is $\mu_{K}$ (Definition 12); and the morphism from concepts to formulas is int (Definition 8).

A context plays the role of a theory extending the subsumption relation and enabling new entailments. Contextualized subsumption can also be seen as a means for extracting knowledge from contexts. Thus, two kinds of knowledge can be extracted: knowledge about context by deduction, and knowledge on 
the domain from which the context is extracted by induction (e.g., generalizing "every bird flies" from bird $\sqsubseteq_{K} f(y)$.

Example 17 (Triv) As the contextualized subsumption is isomorphic to the order on concepts (Theorem 16), it is possible to use the labelled concept lattice (see Figure 1.(b)) to study contextualized subsumption in context $K_{\text {Triv }}$. For instance, as concept 2 is smaller than concept 5 relation $c \sqsubseteq K_{\text {Triv }} b \vee c$ stands, which is already true in $\mathcal{P}$. More generally, it can be seen that all valid subsumptions in $\mathcal{P}$ are retained in contextualized subsumption. Examination of the labelled concept lattice shows that the context adds new valid entailments between formulas: e.g., $c \sqsubseteq_{K_{\text {Triv }}} a \vee b$, because $2 \leq^{c} 4$.

In the following sections, formal contexts will be used to formalize the content of an information system, and the concept lattice (or equivalently, the contextualized subsumption) will be used to organize things. It is the contextualized equivalence relation that gives so many names to things.

\subsection{Feature Context}

Logical languages contain usually infinitely many formulas, whose complexity is unbounded. This is a problem for algorithms that perform a search among formulas (e.g., for automated learning, Ganter and Kuznetsov, 2001). For efficiency and readability of results, we restrict the search space of formulas to a finite subset $F \subseteq \mathcal{L}$ whose elements are called features. Features differ from attributes of standard formal contexts in three ways:

(1) features belong to a fixed logical language and so, have a semantics,

(2) features are automatically ordered according to the subsumption $\sqsubseteq$, and

(3) a newly introduced feature can have a non-empty extent.

It is possible to extract a formal context, with $F$ as the set of attributes, from the logical context: we call it the feature context. This context is not intended to be actually build from the logical context, but it is defined to allow reasoning about the logical context with a coarser grain than the full logic.

Definition 18 (feature context) Let $K=(\mathcal{O},\langle\mathcal{L} ; \sqsubseteq\rangle, d)$ be a logical context, and $F_{K} \subseteq \mathcal{L}$ be a finite set of features, that may depend on $K$. The feature context of $K$ is the formal context $K_{F}=\left(\mathcal{O}, F_{K}, I_{K_{F}}\right)$, where $I_{K_{F}}=\left\{(o, x) \in \mathcal{O} \times F_{K} \mid d(o) \sqsubseteq x\right\}$. We also define description features for any object $o$ by $D_{K_{F}}(o)=\uparrow_{F} d(o)$, where for any $f \in \mathcal{L}$, $\uparrow_{F} f=\{x \in F \mid f \sqsubseteq x\}$. 
The content of $F_{K}$ is not strictly determined but depends on the context $K$. It should contain simple formulas subsuming logical descriptions (in $K$ ), frequently used formulas (in queries), and more generally, every formulas that users expect to see in answers. In fact, $F_{K}$ acts as the vocabulary that a LIS uses in its answers.

Example 19 (Triv) From boolean descriptions (like in context $K_{\text {Triv }}$, see Example 6) one can consider as features the clauses (i.e., disjunctions) of the conjunctive normal form of the descriptions. Thus, the features of context $K_{\text {Triv }}$ would be $F_{K_{\text {Triv }}}=\{a, b, c, a \vee b\}$, and relation $I_{K_{F}}$ would be $\{(x, a),(x, a \vee b),(y, b),(y, a \vee b),(z, c),(z, a \vee b)\}$

From the description given in Example 7, one can extract the following features (amongst others): author contains "Ridoux", year in 1950..2000.

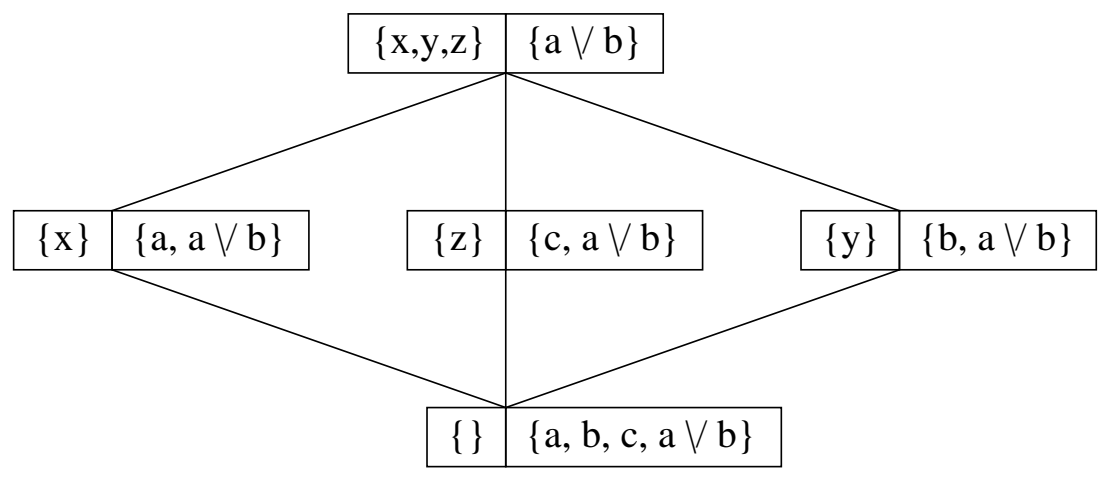

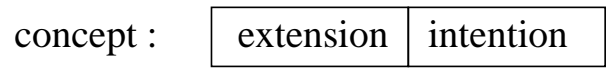

Figure 2. The feature concepts of context $K_{\text {Triv }}$

Feature concepts can be derived from a feature context as for ordinary logical contexts. See for instance Figure 2 for the lattice of feature concepts of context $K_{\text {Triv }}$.

Lemma 20 relates the Galois connections of logical and feature contexts.

\section{Lemma 20 (logical vs. feature Galois connections)} Let $O \subseteq \mathcal{O}, X \subseteq F$,

- $\sigma_{K_{F}}(O)=\uparrow_{F} \sigma_{K}(O)$

- $\tau_{K_{F}}(X)=\tau_{K}(\sqcap X)$.

Theorem 21 shows the existence of a mapping that approximates a logical concept in a feature concept and then defines equivalence classes among logical concepts.

Theorem 21 (approximation of concept) Let $(O, f) \in \mathcal{C}_{K}$ be a logical 
concept. The feature concept generated from $O$ (intent: $\left.\sigma_{K_{F}}(O)\right)$ and the feature concept generated from $f$ (extent: $\tau_{K_{F}}\left(\uparrow_{F} f\right)$ ) are in fact the same feature concept $\left(\tau_{K_{F}}\left(\uparrow_{F} f\right), \uparrow_{F} f\right)$, the smallest concept in $\mathcal{C}_{K_{F}}$ whose extent is larger than or equal to $O$.

\subsection{Sub-context}

It is often useful to reason on a sub-context by restricting the set of objects and the set of features. For instance, the need for views has been recognized in databases (Ullman, 1989) (see also Definition 31).

Definition 22 (sub-context) Given a domain $D \subseteq \mathcal{O}$, restricting the set of objects, and a view $V \subseteq F$, restricting the set of features, we define the sub-context of a feature context $K_{F}$ by the formal context $K_{F}(D, V)=\left(D, V, I_{K_{F}} \cap(D \times V)\right)$.

Example 23 (Triv) We define 4 sub-context of $K_{\text {Triv }}$ (see Example 6).

(1) $D=\mathcal{O}$ and $V=F_{K_{\text {Triv }}} \backslash\{c\}$ :

$I_{K_{F}}=\{(x, a),(x, a \vee b),(y, b),(y, a \vee b),(z, a \vee b)\}$.

(2) $D=\mathcal{O}$ and $V=\{c\}$ :

$I_{K_{F}}=\{(z, c)\}$.

(3) $D=\mathcal{O} \backslash\{z\}$ and $V=F_{K_{T r i v}}$ :

$I_{K_{F}}=\{(x, a),(x, a \vee b),(y, b),(y, a \vee b)\}$.

(4) $D=\mathcal{O} \backslash\{z\}$ and $V=F_{K_{\text {Triv }}} \backslash\{c\}$ :

$I_{K_{F}}=\{(x, a),(x, a \vee b),(y, b),(y, a \vee b)\}$.

Lemma 24 relates the Galois connections of feature contexts and sub-contexts.

Lemma 24 (feature vs. sub-context Galois connection) Let $O \subseteq D$, and $X \subseteq V$,

- $\sigma_{K_{F}(D, V)}(O)=\sigma_{K_{F}}(O) \cap V$,

- $\tau_{K_{F}(D, V)}(X)=\tau_{K_{F}}(X) \cap D$.

Example 25 (Triv) The concept lattices of the four subcontexts of Example 23 are as in Figure 3.

A domain can be specified by the extent $\tau_{K}(q)$ of a formula, i.e., the answers $\tau_{K}(q)$ to a query $q$. A view can be specified as the set of features subsumed by a query $v$, i.e., $\downarrow_{F} v=\{x \in F \mid x \sqsubseteq v\}$. E.g., in the logic presented in Example 7, the formula (author contains "" $\checkmark$ title contains "concept" $\vee$ year in 1900..2000) would select all features of attributes author, title and year, restricted to years in the last 


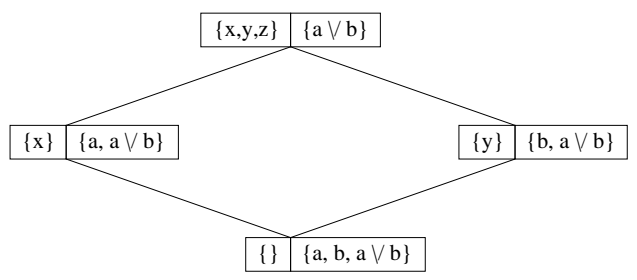

(1)

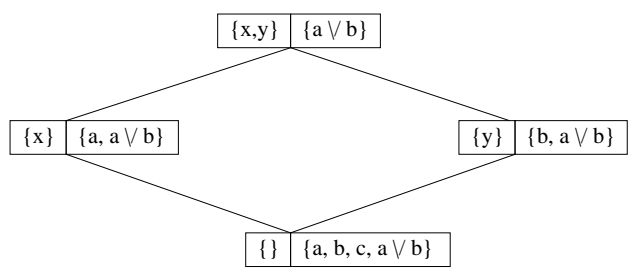

(3)

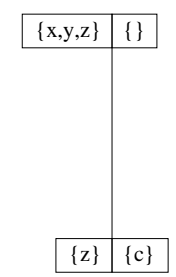

(2)

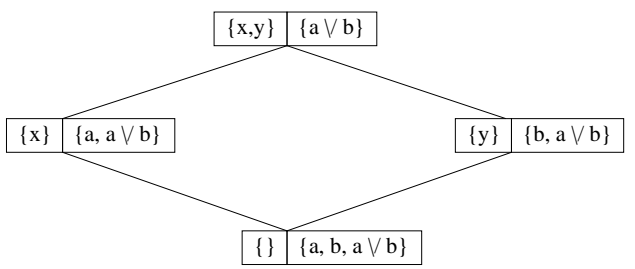

(4)

\begin{tabular}{ll|l|} 
& & \\
& & \\
& extenception & intention \\
\hline
\end{tabular}

Figure 3. Four subconcept lattices of context $K_{\text {Triv }}$

century, and titles that contain the word "concept".

\section{Navigating and Querying in a Logical Context}

\subsection{Navigating vs. Querying}

Information systems offer means for organizing data, and for navigating and querying. Though navigation and querying are not always distinguished because both involve queries and answers, we believe they correspond to very different paradigms of human-machine communication. In fact, the difference can be clarified using the intent/extent duality.

Navigation implies a notion of place, and of a relation between places (e.g., file system directories, and links or subdirectory relations). Through navigation, a user may ask for the content of a place, or ask for related places. The ability to ask for related places implies that answers in the navigation-based paradigm belong to the same language as queries. In terms of the intent/extent duality, a query is an intent, and answers are extents for the content part, and intents for the related places.

In very casual terms, we consider navigation with possibly "no road-map", i.e., no a priori overview of the country. Related places form simply the land- 
scape from a given place as shown by a "viewpoint indicator". However, our proposal is compatible with any kind of a priori knowledge from the user.

With querying, answers are extents only. A simulation of navigation is still possible, but forces the user to infer what could be a better query from the unsatisfactory answer to a previous query; i.e., infer an intent from an extent. This is difficult because there is no simple relation between a variation in the query, and the corresponding variation in the answer. The experience shows that facing a query whose extent is too vast, a user may try to refine it, but the resulting extent will often be either almost as vast as the former or much too small. In the first case, the query lacks of precision (i.e., number of relevant items in the answer divided by total number of items in the answer), whereas in the second case, the query recall (i.e., number of relevant items in the answer divided by number of relevant items in the system) is too low.

Godin et al. (1993) and Lindig (1995) have shown that Formal Concept Analysis is a good candidate for reconciliating navigation and querying. We follow this opinion, but we believe that care must be taken to make formal contexts as close to the description languages of the end-users, and we have proposed Logical Concept Analysis (LCA) where formal descriptions are logical formulas instead of being sets of attributes (Section 2.1).

Our goal in this section is to show how a form of navigation and querying can be defined, so that a user who knows neither the content of a Logical Information System, nor the logic of its descriptions, can navigate in it and discover the parts of the contents and the parts of the logic that are relevant to his quest. Note that a more expert user may know better and may navigate more directly to his goal, but since almost everybody has his shortcomings, the no-knowledge assumption is the safest one to do.

\subsection{A Logical Information System}

In this section, we will insist on navigation tools, and will delay the management of a logical context (e.g., creating, updating objects) until Section 4.

A LIS needs a user interface. We will formalize a shell-based interface, though this is not the most modern thing to do. This is because we believe that shell interfaces (like in UNIX or MS-DOS) are familiar to many of us, and because this abstraction level exposes properly the dialogue of queries and answers. A higher-level interface like a graphical one would hide it, whereas lower-level interfaces, like a file system, would expose irrelevant details. However, nothing prevents one to give a graphical interface to a LIS, or to implement it as a file system. 
The shell commands are those of the UNIX shell, reinterpreted in the LIS framework. Main changes are the replacement of paths by formulas of $\mathcal{L}$ referring to concepts via mapping $\mu_{K}$, and the use of contextualized subsumption $\sqsubseteq_{K}$. For the rest, commands have essentially the same effects. The correspondence between a UNIX file system and a logical information system is as follows:

$$
\begin{aligned}
\text { UNIX shell } & \longrightarrow \text { LIS shell } \\
\text { file } & \longrightarrow \text { object } \\
\text { path } & \longrightarrow \text { logical formula }
\end{aligned}
$$

absolute name of a file $\longrightarrow$ object description

$$
\begin{aligned}
\text { directory } & \longrightarrow \text { formula/concept } \\
\text { root } & \longrightarrow \text { formula } T / \text { concept } T^{c}
\end{aligned}
$$

working directory $\longrightarrow$ working query/concept

Navigation commands cd, ls, and pwd are defined in Section 3.3; and the querying command $\mathrm{Is}-\mathrm{R}$ is defined in Section 3.4. Creation and update commands touch, mkdir, rm, mv, and cp will be defined in Sections 4.1 and 4.2.

\subsection{Navigating in a Logical Context}

Once objects have been logically described and recorded in a logical context $K=(\mathcal{O}, \mathcal{L}, d)$, one wants to retrieve them. One way to do this is navigating in the context. As already said, this way of searching is particularly useful in a context where the logic or the content are unknown. The aim of navigation is thus to guide the user from a current place to a target place, which contains the object(s) of interest. For this, a LIS offers to the user 3 basic operations (the corresponding UNIX-like command names are placed between parenthesis):

(1) to ask to LIS what is the current place (command pwd),

(2) to go in a certain "place" (command cd place),

(3) to ask to LIS effective ways towards other "places" (command 1s).

\subsubsection{Places as formal concepts}

In a hierarchical file system, a "place" is a directory. In our case, a "place" is a formal concept, which can be seen as a coherent set of objects (extent) and 
properties (intent) (see Definition 8). In large contexts, concepts cannot be referred to by enunciating either their extent or their intent, because both are generally too large. Formulas of the logic $\mathcal{L}$ can play this role because every formula refers to a concept through the labelling map $\mu$ (see Definition 12), and every concept is referred to by one or several formulas, which are often much concise than its intent. For instance in Figure 1, one can see that $c$ is a concise name for the concept $(\{z\}, c \wedge(a \vee b))$.

We now describe the 3 navigation operations listed above. First of all, going from place to place implies to remember the current place, which corresponds to the working directory. In a LIS, we introduce the working query, wq, and the working concept, $w c:=\mu_{K}(w q)$; we say that $w q$ refers to $w c$. This working query is taken into account in the interpretation of most LIS commands, and it is initialized to the formula $T$, which refers to the concept whose extent is the set of all objects. Command pwd displays the working query to the user.

The second navigation operation, command cd, takes as argument a query formula $q$ saying in which place to go, and it changes the working query accordingly. We call $l_{w q}$ (i.e., elaboration of $w q$ ) the mapping that associates to the query $q$ a new working query according to the current working query $w q$. The query $q$ can be seen as a link between the current and the new working query. Usually, cd is used to refine the working concept, i.e., to select a subset of its extent. In this case, the mapping $l_{w q}$ is defined by $l_{w q}(q):=w q \sqcap q$, which is equivalently characterized by

$$
\mu_{K}\left(l_{w q}(q)\right)={ }^{c} w c \wedge^{c} \mu_{K}(q) \text { and } \tau_{K}\left(l_{w q}(q)\right)=\tau_{K}(w q) \cap \tau_{K}(q) .
$$

However, it is useful to allow for other interpretations of the query argument. For instance, we can allow for the distinction between relative and absolute queries, similarly to relative and absolute paths in file systems. The previous definition of the mapping $l_{w q}$ concerns relative queries, but can be extended to handle absolute queries by $l_{w q}(/ q):=q$, where '/' denotes the absolute interpretation of queries. This allows to forget the working query. We can also imagine less usual interpretations of queries like $l_{w q}(\mid q):=w q \sqcup q$. Finally, the special argument .. for the command cd enables to go back in the history of visited queries/concepts. This works much like the "Back" button in Web browsers.

The last navigation operation, command $1 \mathbf{s}$, is intended to guide the user towards his goal. More precisely, it must suggest some relevant links that could act as queries for the command cd to refine the working query. These links are formulas of $\mathcal{L}$. A set of links given by ls should be finite, of course (whereas $\mathcal{L}$ is usually infinite), even small if possible, and complete for navigation (i.e., each object of the context must be accessible by navigating from $T^{c}$ ). 


\subsubsection{Navigation Links}

The following notion of link corresponds to the case where the elaboration mapping satisfies $l_{w q}(q)=w q \sqcap q$. To avoid to go in a concept whose extent is empty (a dead-end), we must impose the following condition on a link $x$ : $\tau_{K}(w q \sqcap x) \neq \emptyset$. Furthermore, to avoid to go in a concept whose extent is equal to the extent of $w q$ (a false start), we must impose this other condition: $\tau_{K}(w q \sqcap x) \neq \tau_{K}(w q)$. These conditions state that the extent of the new working query must be strictly between the empty set and the extent of the current working query. This characterizes relevant links (Lindig, 1995).

Now, as $\mathcal{L}$ is a too wide search space (it is often infinite), we will consider a finite set of features $F \subseteq \mathcal{L}$ in a context $K$ in which links are selected.

Furthermore, we retain only greatest links (in the subsumption order) as they correspond to smallest navigation steps. Indeed, recall that each link is a suggestion to the user. Therefore, if the link year in 1990 . 2010 is suggested, it is not worth suggesting the link year $=2000$, because the former subsumes the latter. Following this principle, every conjunctive formula $x \sqcap y$ can be excluded from the search space for links, and so, of the set of features, because it is redundant with $x$ and $y$. Thus, a way to build the set of features is to split object descriptions on outermost conjunctions (i.e., $a \wedge b$ is split into $a$ and $b$, whereas $(c \wedge d) \vee e$ cannot be split this way).

However, to limit the number of links, it is useful to also extract abstracted form of these features, so as to allow a still more progressive navigation. For instance, the system would suggest the successive links title, title contains "System", and then title is "Logical Information Systems"; instead of directly suggesting the latter. Only this latter feature appears explicitly in the description. The others are abstractions of it, and plays a role of "factorization" among links.

We now summarize this part by defining the set of links in a given context and working query.

Definition 26 (Navigation links) Let $K=(\mathcal{O}, \mathcal{L}, d)$ be a context. The set of navigation links for every working query $w q \in \mathcal{L}$ is defined by

$$
\operatorname{Link}_{K}(w q):=\operatorname{Max}_{\sqsubseteq}\left\{x \in F_{K} \mid \emptyset \neq \tau_{K}(w q \sqcap x) \neq \tau_{K}(w q)\right\} .
$$

\subsubsection{Local Objects and Navigation Completeness}

As navigation aims at finding objects, command 1s must not only suggest some links to other places, but also present the objects belonging to the current place, called the objects of $w q$ or the local objects. We define a local object 
as an object that is in the current place, but in no place reachable through a link.

Definition 27 (local object) Let $K=(\mathcal{O}, \mathcal{L}, d)$ be a context. The set of local objects is defined for every working query $w q \in \mathcal{L}$ by

$$
\operatorname{Local}_{K}(w q):=\tau_{K}(w q) \backslash \bigcup_{x \in \operatorname{Link}_{K}(w q)} \tau_{K}(x)
$$

Given a place, there can be no local object, but there can also be several objects in some place. The less local objects there are, the better it is for navigation. More precisely, if two local objects have non-equivalent descriptions, it should be possible to make this difference appear in the links. Thus, the choice the user has to do is intentional (between one or several logical links) rather than extensional (between several objects). This idea is formalized as the completeness of navigation.

Definition 28 (navigation completeness) Let $K=(\mathcal{O}, \mathcal{L}, d)$ be a context. Navigation is complete in $K$ if and only if for every working query $w q \in \mathcal{L}$, the following holds

$$
\forall o, o^{\prime} \in \operatorname{Local}_{K}(w q): d(o) \equiv d\left(o^{\prime}\right)
$$

This completeness can be guaranteed by ensuring that the set of features $F_{K}$ is such that if two objects have non-equivalent descriptions there exists a feature that is satisfied by one object and not by the other.

Theorem 29 (navigation completeness) A necessary and sufficient condition for navigation completeness in a context $K$ is that for every objects $o, o^{\prime}$

$$
d(o) \not \equiv d\left(o^{\prime}\right) \Rightarrow \exists x \in F_{K}: d(o) \sqsubseteq x \Leftrightarrow d\left(o^{\prime}\right) \nsubseteq x .
$$

In the case where all objects have different descriptions, there is never more than one local object. This must be compared to Web querying where the number of objects returned in response to a query is generally large. This is because with navigation, non-local objects are hidden behind the intentional properties that enable to distinguish these objects. It is the end-user who selects an intentional property to reveal its content.

Another interesting thing to notice is that the working query can be, and often is, much shorter than the whole description of the local object (which is also the intent of the working concept), as in the following example where the first formula is contextually equivalent (in context Bib, see Example 7) to the 
second one for accessing the object.

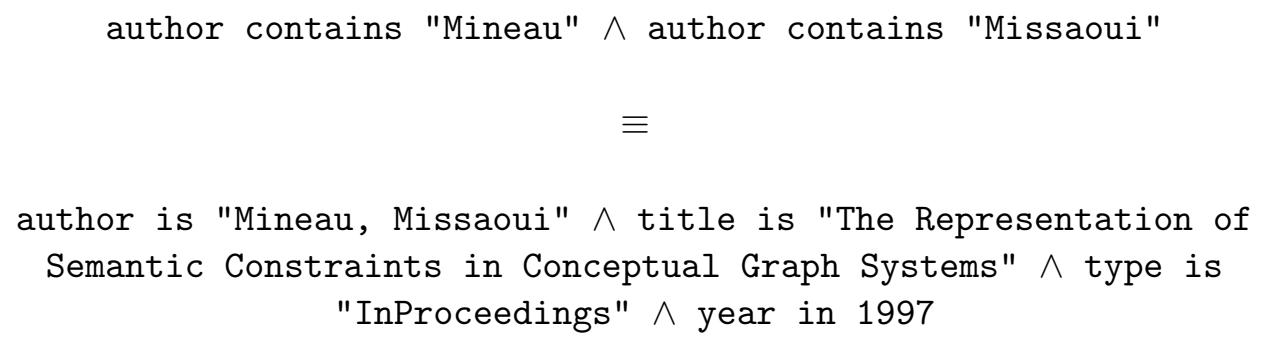

\subsubsection{Links and Views}

Even if the set of links is restricted to relevant and greatest ones among features, it appears in practice that it is too large and heterogeneous. For instance, in the context Bib (see Example 7), the set of links is a mix of author names, title words, etc. Our idea is to abstract the set of all author names (features author contains ...) by a single feature author (meaning "the attribute author is defined"). This feature is not useful for selecting objects, but it is useful to select links about the attribute author. We call this kind of features views as they present the navigation from a "point of view". We introduce a working view $w v$, similar to the working query, under which links must be searched for. For instance, if the working view is (author), links will be author names. We take it into account in the definition of navigation links, where the relation $x \sqsubset w v$ denotes a strict subsumption (i.e., $x \sqsubseteq w v$ and $w v \nsubseteq x)$.

Definition 30 (Navigation links with views) Let $K=(\mathcal{O}, \mathcal{L}, d)$ be $a$ context. For every working query $w q \in \mathcal{L}$ and every working view $w v \in \mathcal{L}$, the set of navigation links is defined by

$$
\operatorname{Link}_{K}(w q, w v):=\operatorname{Max}_{\sqsubseteq}\left\{x \in F_{K} \mid x \sqsubset w v, \emptyset \neq \tau_{K}(w q \sqcap x) \neq \tau_{K}(w q)\right\} .
$$

As a link is a variation of the working query that restricts the current extent, one defines a view as a variation of the working view that restricts the set of links. Moreover, if a view is not also a link, it must subsume at least two links. Indeed, if some view hides only one link, it is worth presenting the link directly. Finally, we define a set of links and views where views can be understood as summaries of sets of links, whose selection by the user allows him to see these underlying links in a narrower view.

Definition 31 (Navigation links and views) Let $K=(\mathcal{O}, \mathcal{L}, d)$ be a context. The set of navigation links and views for every working query $w q \in \mathcal{L}$ and every working view $w v \in \mathcal{L}$ is defined by $\|E\|$ denotes the cardinality of 
a set $E$ )

$$
\begin{array}{r}
L V_{K}(w q, w v):=\operatorname{Max}_{\sqsubseteq}\left\{x \in F_{K} \mid x \sqsubset w v, \quad \emptyset \neq \tau_{K}(w q \sqcap x) \neq \tau_{K}(w q)\right. \\
\text { or } \left.\left\|\operatorname{Link}_{K}(w q, w v \sqcap x)\right\| \geq 2\right\} .
\end{array}
$$

To summarize, the view-based variant of command ls takes as argument a view $v$, sets the working view to $l_{w v}(v)$ (where $l_{w v}$ works similarly to $l_{w q}$ ), shows the local objects if they exist, displays each link or view $x$ of the set $L V_{K}(w q, w v)$ along with the size of its selected extent $\tau_{K}(w q \sqcap x)$, and finally displays the size of the working extent $\tau_{K}(w q)$. Links and views are distinguished according to their cardinality compared to the size of the working query; views simply have the same size as the working concept, whereas links have strictly smaller sizes.

\subsubsection{User/LIS Dialogue}

We now show how commands cd and ls compose a rather natural dialogue between the user and LIS. The user can refine the working concept with command cd, and asks for suggested links and views with the command 1s. LIS displays to the user relevant links for forthcoming cd's, and relevant views for forthcoming 1s's. With commands cd and 1s, and links and views, both the user and a LIS can assert facts and ask questions;

- Command cd and links are assertions from the user and LIS.

user: cd kind (i.e., "I want this kind of object!"),

LIS: links to kind (i.e., "I have this kind of object!").

- Command is and views are questions from the user and LIS.

user: Is kind (i.e., "What kind of object do you have?"),

LIS: view kind (i.e., "What kind of object do you want?").

It should also be noticed that both the user and LIS can answer to questions both by assertions and by questions.

Example 32 (Bib) A complete example of a dialogue is given in Table 1. The left part of this table shows what is really displayed by our prototype, and the right part is an english translation of the dialogue. Notice that this translation is rather systematic and could be made automatic. $(n)$ is the prompt for the $n$-th query from the user. On the 2nd query, the question of the user is so open, that LIS only answers by questions. On the 3rd query, the user replies to one of these questions (title) by an assertion; but on the 4 th query, he sends back to LIS another of these questions (author) to get some relevant suggestions. On the 5th query, he just selects a suggested author, "Wille", and then gets his co-authors on Concept Analysis with the 6th query. On the 
7th query, he selects a co-author and finally finds an object at the 8th query.

Table 1

Example of User/LIS Dialogue in context Bib.

\begin{tabular}{|c|c|}
\hline (1) pwd & (1) What is currently selected? \\
\hline 1 & All objects. \\
\hline (2) $1 \mathrm{~s}$ & (2) What do you have? \\
\hline 209 type & What kind of type do you want? \\
\hline 209 author & What kind of author do you want? \\
\hline 209 year & What kind of year do you want? \\
\hline 209 title & What kind of title do you want? \\
\hline 209 object(s) & 209 objects are currently selected. \\
\hline (3) cd title contains "Concept A" & (3) I want objects whose title contains "Concept A"! \\
\hline (4) Is author & (4) What kind of author do you have (for this)? \\
\hline 1 author contains "Mineau" & I have 1 object with author "Mineau"! \\
\hline 1 author contains "Lehmann" & I have 1 object with author "Lehmann"! \\
\hline 1 author contains "Stumme" & I have 1 object with author "Stumme"! \\
\hline 1 author contains "Prediger" & I have 1 object with author "Prediger"! \\
\hline 3 author contains "Wille" & I have 3 objects with author "Wille"! \\
\hline $4 \operatorname{object}(\mathrm{s})$ & 4 objects are currently selected. \\
\hline (5) cd author contains "Wille" & (5) I want objects with author "Wille"! \\
\hline (6) Is & (6) What kind of author do you have (now)? \\
\hline 1 author contains "Mineau" & I have 1 object with author "Mineau"! \\
\hline 1 author contains "Lehmann" & I have 1 object with author "Lehmann"! \\
\hline 1 author contains "Stumme" & I have 1 object with author "Stumme"! \\
\hline $\begin{array}{l}3 \text { author contains "Wille" } \\
3 \text { object(s) }\end{array}$ & $\begin{array}{l}\text { What kind of author "Wille" do you want? } \\
3 \text { objects are currently selected. }\end{array}$ \\
\hline $\begin{array}{l}\text { (7) cd author contains "Mineau" } \\
\text { (8) Is }\end{array}$ & $\begin{array}{l}\text { (7) I want objects with author "Mineau"! } \\
\text { (8) What do you have? }\end{array}$ \\
\hline \multicolumn{2}{|c|}{$\begin{array}{l}\text { \#200 Mineau, Stumme, Wille. Conceptual Structures Represented by Conceptual Graphs } \\
\text { and Formal Concept Analysis. INPROC, } 1999 .\end{array}$} \\
\hline $1 \operatorname{object}(\mathrm{s})$ & 1 object is currently selected. \\
\hline (9) pwd & (9) What is currently selected? \\
\hline author contains "Wille" $\wedge$ & Objects with authors "Wille" \\
\hline author contains "Mineau" $\wedge$ & and "Mineau", \\
\hline title contains "Concept A" & and whose title contains "Concept A". \\
\hline
\end{tabular}

\subsection{Querying a Logical Context}

Extensional queries, as in data-bases or some Web browsers like Google, can be submitted to a logical information system using the $-\mathrm{R}$ option with command 1 s. The answer to query 1 s $-\mathrm{R} q$ is simply $\tau_{K}\left(l_{w q}(q)\right)$, i.e., the extent 
of the concept referred to by $l_{w q}(q)$ (see Section 3.3).

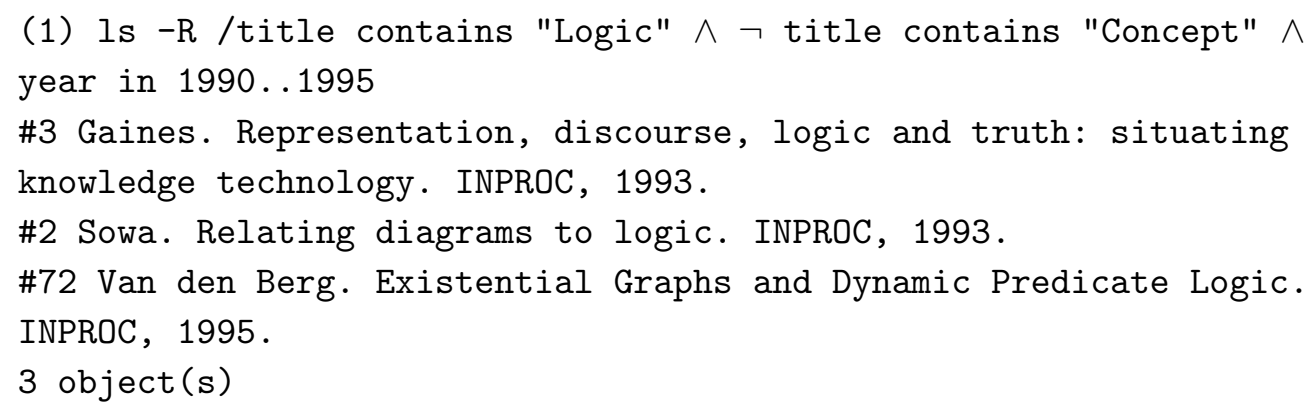

\section{Creating and Updating a Logical Context}

Whereas much has been said on the construction of concept lattices (Kuznetsov and Objedkov, 2001), the construction of contexts is often left in the background. The construction process can fall into two categories: off-line and on-line. In the off-line case, the context is built once for all after the data have been gathered and the problem is to find an object description language appropriate to the intended analysis. The typical application of this category is the analysis of surveys. In the on-line case, the context is built progressively along the arrival of data and a problem is to properly describe new objects at the time they arrive. Information systems are the typical application of this category, but this is not the mainstream approach to using Concept Analysis.

Hypothesis 1 (on-line construction) We consider here only the on-line case, as we focus on information systems. For each new piece of data that arrives, an object is created, and added to the context, with this piece of data as content.

We propose that the description given to an object is two-parts. The first part, the intrinsic description, is automatically extracted from the object content, and depends on the kind of content and on the logic of the application. For instance, let us consider that objects are incoming e-mail messages. In this application, the building of the context is clearly on-line; and possible components of the intrinsic description are the from, to, and subject fields.

The second part, the extrinsic description, is manually assigned by users according to personal intentions and preferences. We must consider there are no known rules to infer extrinsic properties, as if the contrary holds they could be integrated in the intrinsic description. In a usual e-mail application, extrinsic properties are managed by storing e-mail messages in different folders accord- 
ing to personal needs. However, extrinsic properties need not be organized in a hierarchical relation as folders often are.

\subsection{Creating a Logical Context}

The objects we want to represent in a context are often defined in the "world" by a content. We want to describe these objects both by an intrinsic properties (automatically extracted from their contents), and by extrinsic properties (manually assigned by users). All these elements are the context data, from which a logical context can be built.

Definition 33 (context data) Context data are defined as a 6-tuple $D=\left(\mathcal{O}, \mathcal{C}, \mathcal{L}, c, d_{i}, d_{e}\right)$, where

- $\mathcal{O}$ is a set of objects,

- $\mathcal{C}$ is the domain of contents,

- $\mathcal{L}$ is a logic,

- $c \in \mathcal{O} \rightarrow \mathcal{C}$ maps every object to its content,

- $d_{i} \in \mathcal{C} \rightarrow \mathcal{L}$ extracts an intrinsic description from every content,

- $d_{e} \in \mathcal{O} \rightarrow \mathcal{L}$ maps every object to its extrinsic description.

The building of a context from its data consists in keeping the set of objects and the logic, and composing a description that maps every object to its intrinsic description "plus" its extrinsic description. This "plus" denotes an update operation $\diamond$ that we present in more details in Section 4.2.

Definition 34 (context building) Let $D=\left(\mathcal{O}, \mathcal{C}, \mathcal{L}, c, d_{i}, d_{e}\right)$ be context data. The context built from data $D$ is defined as

$$
K(D)=(\mathcal{O}, \mathcal{L}, d), \text { where for every } o \in \mathcal{O}, d(o)=d_{i}(c(o)) \diamond d_{e}(o) .
$$

Example 35 (E-Mail) In this section, we consider that objects are e-mail messages. An example of a simplified e-mail message content is:

From: Alice@paris.fr

To: Bob@berlin.de, Chloe@madrid.es

Date: 2 May 2002, 14:52

Subject: Hello world!

When do you come in Paris?

See you,

Alice

From such a content, an intrinsic description that retains only fields from, 
to, and subject can be extracted and formulated in logic $\mathcal{P}_{v}$ (see Example 7). This is only a matter of choice, as other fields could be taken into account. In particular, the message body would certainly be useful in a real application, but it is not necessary to our explanations. The intrinsic description we obtain from the above content is the following formula.

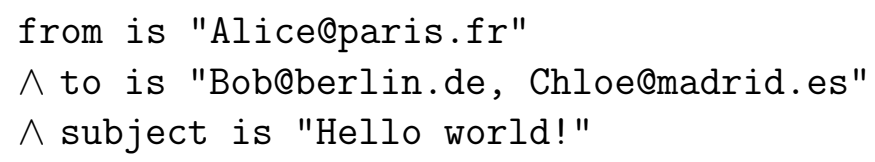

Then, the user (e.g., Bob) can add his personal comments by formulating an extrinsic description. For instance, the extrinsic description

personal $\wedge \neg$ spam

means the message is "personal" (opposite of "professional"), and is not a "spam". The expected result of updating the intrinsic description with the extrinsic description is

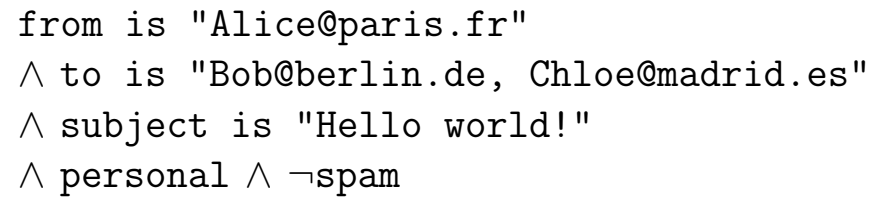

The LIS shell commands for creating new entries in a context are mkdir and touch. Command mkdir creates a new feature. For instance, mkdir subject begins with "H" introduces a finer feature than those that are obtained by simply splitting conjunctions. Command touch simply creates an object with empty content at some place designated by a formula. However, objects are normally created by applications that give objects a content (and so, an intrinsic description) and an extrinsic description, which is usually based on the working query.

\subsection{Updating a Logical Context}

From the definition of context data (see Definition 33), a logical context can be updated in 4 ways:

(1) addition of an object with its initial content and extrinsic description (commands touch, cp, and applications),

(2) update of the content of an object (applications),

(3) update of the extrinsic description of an object (command mv), and

(4) deletion of an object (command $\mathrm{rm}$ ). 
The only difficulty that arises from these operations is the update operation, which is used to change an extrinsic description in an incremental way (i.e., without completely redefining it), and to compose intrinsic and extrinsic descriptions. A naive solution would be to manually change extrinsic descriptions, and to directly integrate them in intrinsic descriptions. However, this has two important drawbacks. First, the intrinsic description changes with the contents and so, it is not a persistent support for extrinsic properties. Second, practice shows that one often wishes to express an update operation for a set of objects in a single command: for instance, add property "personal" (personal) to all e-mail messages sent from "Alice" (i.e., from contains "Alice").

We give now a specification of such an operation, as it is given by Herzig and Rifi (Herzig and Rifi, 1999). This puts constraints on what a logical update operation should be.

Definition 36 (update) Let $\mathcal{L}=(L, \sqsubseteq, \sqcap, \sqcup, \top, \perp)$ be a logic. The result of updating a description $d \in L$ by an entry $e \in L$ is the result of an operation $d \diamond e$, which must satisfy the following postulates:

(1) $(H R) d \diamond e \sqsubseteq e$;

(2) $(H R) d \sqcap e \sqsubseteq d \diamond e$;

(3) $(H R) d \diamond \top \equiv d$;

(4) (HR) $d \nsubseteq \perp$ and $e \nsubseteq \perp$ implies $d \diamond e \nsubseteq \perp$;

(5) (HR) for every $d^{\prime} \equiv d, d^{\prime} \diamond e \equiv d \diamond e$;

(6) (HR) for every $e^{\prime} \equiv e, d \diamond e^{\prime} \equiv d \diamond e$;

(7) (HR) for every $d_{1}, d_{2} \in L$ such that $d \equiv d_{1} \sqcup d_{2}, d \diamond e \equiv\left(d_{1} \diamond e\right) \sqcup\left(d_{2} \diamond e\right)$;

(8) (involution) $(d \diamond e) \diamond e \equiv d \diamond e$.

All postulates are from Herzig and Rifi, except postulate 8. Postulate 1 means that the entry must always be taken into account, and so, satisfied in the result. Postulate 2 adds that everything satisfied in the result comes from either the description $d$ or the entry $e$. In the case where the entry is the tautology, i.e., brings no information, postulate 3 states that the description must be kept unchanged. Postulate 4 forces the result to be consistent, unless the description or the entry is already inconsistent. Postulates 5 and 6 mean that results must not depend on the syntax of formulas. Finally, postulate 7 says that operation $\diamond$ must be distributive for disjunction, and postulate 8 says that it must be an involution.

Herzig and Rifi (1999) present an update operation for propositional logic with dependencies between atoms, denoted by $W S S \downarrow^{d e p}$, that satisfies all postulates in Definition 36. In logic, dependency between atoms is subsumption.

Example 37 (Bib) Logic $\mathcal{P}_{v}$ is a propositional logic, whose usual atoms are replaced by valued attributes. This means that we must consider dependencies between such atoms. For instance, the atom 
from is "Alice@paris.fr" implies the atom from contains "Alice", contradicts the atom from is "Bob@berlin.de", but is independent from the atom subject contains "Hello".

The LIS shell commands rm, mv and $\mathrm{cp}$ perform LIS updates. Command $\mathrm{rm} \mathrm{q}$ suppresses the local object of $l_{w q}(q)$ (if it exists, see Section 3.3.3). Command mv moves a file from a place to another: $\mathrm{mv} q \mathrm{q}$ moves the local object $o$ of $l_{w q}(q)$ in concept $\mu(d(o) \diamond e)$. Similarly, cp q e creates a new copy of the local object in the same concept. With option $-r$, every object of the extent of $l_{w q}(q)$ is concerned, instead of only the local object.

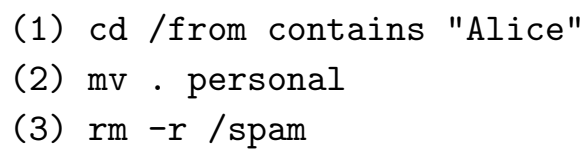

The move command (line 2) adds the feature personal to the local object of the working query from contains "Alice". The remove command (line 3) deletes all objects described as spam.

Contents can also be changed by applications. This changes indirectly descriptions (their intrinsic parts), but the ensuing reorganization of the formal concept lattice is automatic and transparent. In fact, it costs not so much since the concept lattice is not actually represented (see Section 6).

\section{Data-mining, automated updating and learning}

Previous sections 3 and 4 present the core operations of a LIS. More operations can be defined. Some are derived from core operations, like a form of datamining, and others are disjoint from the core but can be added to it.

\subsection{Data-mining}

The definition of links (see Definitions 3.3.2 and following) is a specific case of Knowledge Discovery in a formal context. It can be generalized to recover more classical KD operations like machine-learning through the computation of necessary or sufficient properties (modulo some confidence), or data-mining through association rules. Indeed, CA has been often applied in domains such as data-analysis, data mining, and learning.

Data-analysis consists in structuring data in order to help their understanding. These data are often received as tables or relations and structured by partitions, hierarchies, or lattices. With CA, formal contexts (binary relations 
between objects and attributes) are structured in concept lattices (Ganter and Wille, 1999). This is applied for instance in software engineering for configuration analysis (Krone and Snelting, 1994). Data-mining is used to extract properties from large amounts of data. These properties are association rules satisfied (exactly or approximately) by the data. This is analogous to implications between attributes in FCA (see Ganter and Wille, 1999, p. 79), and to contextualized subsumption in LCA (see Section 2.4). Unsupervised learning is similar to data-analysis in the sense that one tries to discover some properties, and to understand some data, whereas supervised learning is similar to data-mining as some rules are searched to explain a target property using known properties. For instance, Kuznetsov applied CA to the learning of a positive/negative property from positive and negative instances (Kuznetsov, 1999).

The aim of this section is to show that these features of Knowledge Discovery (KD) can be incorporated in LIS, and how.

A context $K$ plays the role of a theory by extending the subsumption relation and enabling new entailments (e.g., bird $\sqsubseteq_{K} f l y$ when every bird flies in the context). All these contextual entailments are gathered with logical entailments to form the contextualized logic, which is thus a means for extracting some knowledge from the context. Two kinds of knowledge can be extracted: knowledge about the context by deduction ("Every bird of this context do fly"), and knowledge about the domain (which the context belongs to) by induction ("Every bird of the domain may fly").

Concept lattices produced by data-analysis are isomorphic to contextualized logics (see Theorem 16). Associations rules produced by data-mining or supervised learning match the contextualized subsumption relation, possibly qualified by a confidence defined by $\operatorname{con} f\left(f \sqsubseteq_{K} g\right)=\frac{\left\|\tau_{K}(f) \cap \tau_{K}(g)\right\|}{\left\|\tau_{K}(f)\right\|}$ and a support defined by $\operatorname{supp}\left(f \sqsubseteq_{K} g\right)=\left\|\tau_{K}(f) \cap \tau_{K}(g)\right\|$.

Considering two properties $f, g \in \mathcal{L}$, their contextual relation is determined by the cardinalities of 3 sets of objects: $\pi_{K}^{l}(f, g):=\left\|\tau_{K}(f) \backslash \tau_{K}(g)\right\|$, $\pi_{K}^{c}(f, g):=\left\|\tau_{K}(f) \cap \tau_{K}(g)\right\|$ and $\pi_{K}^{r}(f, g):=\left\|\tau_{K}(g) \backslash \tau_{K}(f)\right\|$. For instance, $f$ contextually entails $g$ if and only if $\pi_{K}^{l}(f, g)=0, f$ and $g$ are contextually separated if and only if $\pi_{K}^{c}(f, g)=0$, or $x$ is a link of $w q$ (see Section 3.3.2) if and only if $\pi_{K}^{c}(x, w q) \neq 0$ and $\pi_{K}^{r}(x, w q) \neq 0$. Note that the superscripts, $l, c$, and $r$ refer to the left, center, and right part of the following Venn diagram:

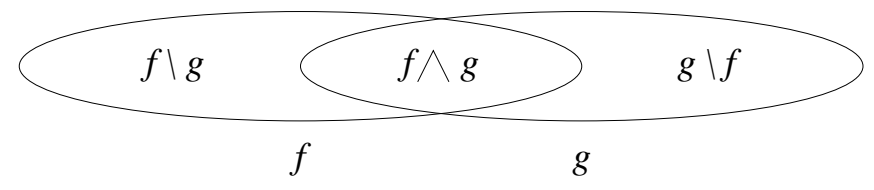


So, the procedure that computes links for navigating in a LIS can be generalized to compute necessary or sufficient conditions, association rules, and links, only by specifying constraints on $\pi_{K}^{c}, \pi_{K}^{r}$, and $\pi_{K}^{l}$. Observe that $\operatorname{con} f\left(f \sqsubseteq_{K} g\right)=\frac{\pi_{K}^{c}(f, g)}{\pi_{K}^{l}(f, g)+\pi_{K}^{c}(f, g)}$, and that $\operatorname{supp}\left(f \sqsubseteq_{K} g\right)=\pi_{K}^{c}(f, g)$.

Definition 38 (Data-mining) Let $K=(\mathcal{O}, \mathcal{L}, d)$ be a context. For every working query $w q \in \mathcal{L}$ and working view $w v \in \mathcal{L}$ a set of facts is defined by

$$
\operatorname{Fact}_{K}(w q, w v):=\operatorname{Max}_{\sqsubseteq}\left\{\begin{array}{r}
(x, \operatorname{rank}(x, w q)) \in F_{K} \times \operatorname{Rank} \\
\mid x \sqsubset w v, \operatorname{property}(x, w q)
\end{array}\right\},
$$

where Rank is a domain of ranking values, and rank and property are an application and a predicate defined using $\pi_{K}^{c}, \pi_{K}^{r}$, and $\pi_{K}^{l}$.

\section{Example 39 (Generalized navigation)}

\section{Links: Consider}

$\operatorname{rank}(x, w q)=\pi_{K}^{c}(x, w q)$ and $\operatorname{property}(x, w q)=\pi_{K}^{c}(x, w q)>0$.

Then, Fact $_{K}(w q, w v)$ computes all links from working query wq under view wv.

Necessary conditions: Consider

$$
\operatorname{rank}(x, w q)=\operatorname{conf}(w q \sqsubseteq K x)
$$

and $\operatorname{property}(x, w q)=\operatorname{conf}\left(w q \sqsubseteq_{K} x\right) \geq \operatorname{con} f_{\text {min }}$,

where con $f_{\text {min }} \in[0,1]$.

Then, $\operatorname{Fact}_{K}(w q, w v)$ computes all necessary properties to wq (i.e., properties entailed by $w q)$ with a confidence greater than con $f_{\min }$.

\section{Sufficient conditions: Consider}

$$
\operatorname{rank}(x, w q)=\left(\operatorname{supp}\left(x \sqsubseteq_{K} w q\right), \operatorname{conf}\left(x \sqsubseteq_{K} w q\right)\right)
$$

and property $(x, w q)=\operatorname{supp}\left(x \sqsubseteq_{K} w q\right) \geq \operatorname{supp}_{\min } \wedge \operatorname{conf}\left(x \sqsubseteq_{K} w q\right) \geq \operatorname{conf}_{\text {min }}$, where $\operatorname{supp}_{\min }$, con $f_{\text {min }} \in[0,1]$.

Then, $\operatorname{Fact}_{K}(w q, w v)$ computes all sufficient conditions to be in the extent of wq with a support and confidence greater than supp $p_{\min }$ and conf $f_{\text {min }}$.

\subsection{Context Maintenance based on Learning}

A context associates to objects a description that combines automatically extracted properties (intrinsic) and manually assigned ones (extrinsic) (cf. Section 4). The extrinsic properties are expressed by users according to intentions that are often subjective and changing, and that determine the classification and retrieval of objects. So, we believe it is important to assist users in this task through the automatic suggestion of extrinsic properties to be assigned 
and even the discovery of rules to automate these assignments. The principle is to learn from the relationship between extrinsic and intrinsic descriptions of existing objects the extrinsic description of a new object whose intrinsic description is computed from its content. Because of the changing nature of users' intentions, the assistance given in the incremental building of a logical context must be interactive. We present formal principles, and an application to the classification of e-mail messages. Proofs can be found in (Ferré and Ridoux, 2002b).

\subsubsection{Induction through Associative Concepts}

Let us consider the situation where a new object $o^{*}$ is added to a logical context $K=(\mathcal{O}, \mathcal{L}, d)$ along with an intrinsic description $d^{*}\left(o^{*}\right)$ to form a new context $K^{*}=\left(\mathcal{O} \uplus\left\{o^{*}\right\}, \mathcal{L}, d^{*}\right)$ with $d^{*}(o)=d(o)$ for all $o \in \mathcal{O}$. Our aim is to induce from the old context $K$ a set of extrinsic properties $\operatorname{Ind}_{K_{F}}\left(o^{*}\right) \subseteq F$ for the new object.

We first define associative concepts as the concepts of $K$ when one considers only description features of $o^{*}, D_{K_{F}}\left(o^{*}\right)$.

Definition 40 (associative concept) A non-empty concept of the subcontext $K_{F}\left(\mathcal{O}, D_{K_{F}}\left(o^{*}\right)\right)$ is called an associative concept of $o^{*}$ in $K_{F}$. The set of all such associative concepts is denoted by $A C_{K_{F}}\left(o^{*}\right)$.

$A C_{K_{F}}\left(o^{*}\right)$ organizes the feature context $K_{F}$ in a concept lattice (where the empty concept is missing) that is less finely detailed than $\mathcal{C}_{K_{F}}$. However, this coarser concept lattice is relevant to the features of $o^{*}$. Conversely, the finer details in $\mathcal{C}_{K_{F}}$ cannot be expressed with the features of $o^{*}$.

Then, an induced feature can be defined as a feature that contextually subsumes the intent of some associative concepts.

Definition 41 (induced property) We say a feature $x$ is an induced property for $o^{*}$ if and only if there exists an associative concept $c \in A C_{K_{F}}\left(o^{*}\right)$, such that $\sqcap \operatorname{int}(c) \sqsubseteq_{K} x$ or, equivalently, ext $(c) \subseteq \tau_{K}(x)$. Ind ${K_{K}}_{F}\left(o^{*}\right)$ denotes the set of all induced properties of $o^{*}$ in $K_{F}$.

Intuitively, an associative concept $c$ of a new object $o^{*}$ is an already existing concept (for previous objects in $K$ ) that has some similarity with the description of $o^{*}$. When $x \in \operatorname{Ind}_{K_{F}}\left(o^{*}\right)$ is induced from an associative concept $c$, $\operatorname{ext}(c)$ is the support of the induction, and int $(c)$ is the explanation. A given associative concept can induce several features; and a given feature can be induced by several associative concepts, and so, it can have several explanations. Induced features that do not belong to description features are called expected features, which are the features suggested to users as extrinsic properties. 
Example 42 (Triv) Consider $d\left(o^{*}\right)=c \wedge d$, then $D_{K_{F}}\left(o^{*}\right)=\{c, d\}$. $a c=(\{z\},\{c\})$ is an associative concept of $o^{*}$, and $a \vee b$ is a feature of $K_{\text {Triv }}$ (see Figure 2) such that $\operatorname{ext}(a c) \subseteq\{x, y, z\}=\tau_{K_{\text {Triv }}}(a \vee b)$. So, $a \vee b$ is an induced feature.

\subsubsection{Experimentation}

The aim of this section is to present through experimentations the kind of interactions that help a user to assign extrinsic properties to incoming objects, and to gradually automate these assignments (e.g., for filtering spams).

5.2.2.1 Filtering spams We consider here the assisted filtering of spams. The following display shows the initial description of a new (non-solicited) email message, with its expected features. The context on which the induction of expected features is based is made of 200 e-mail messages.

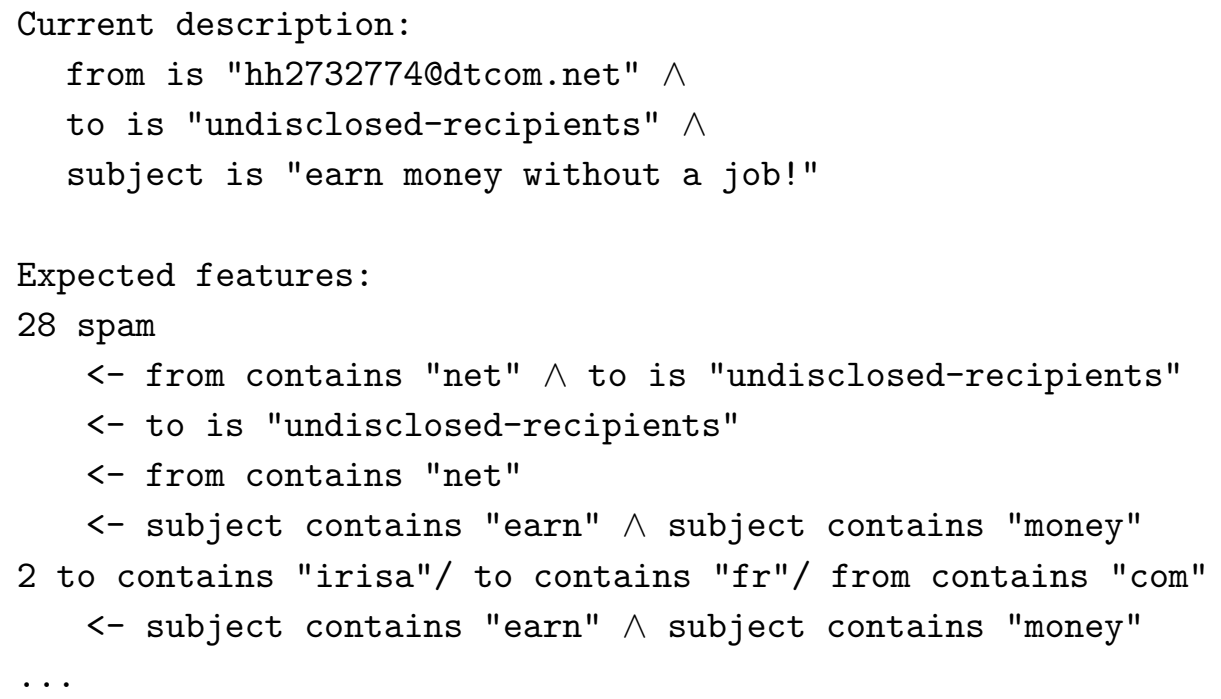

We can see that, whereas the from and subject field are new, several features are induced. This is possible because message fields are split into words, which enables to find common features between the new object and existing ones: e.g., from contains "net", subject contains "money", to is "undisclosed-recipients".

We also see that the above message is well-recognized as a spam, and this feature is even the most strongly induced one with several explanations and a weight of 28 supporting objects. These explanations suggest some rules such as "every e-mail message sent to undisclosed-recipients is a spam", or "every e-mail message whose subject contains words earn and money is a spam". 
It is up to the user to validate these explanations, and to make them automatic rules, or to be cautious, and to consider them only as hints.

Figure 4 shows the filtering rate of spams during the incremental building of an e-mail context. The dashed line represents the rate of well-classified messages (as spam or non-spam) at the n-th insertion. The solid line represents the rate of classified messages (well or not). So, the part between the two lines represents badly classified messages (e.g., spam classified as non-spam), and the part above the solid line represents non-classified messages. This plot shows that after a transient phase of about 50 messages, the rate of well classified messages steadily reaches $85 \%$, and there are nearly no bad classification.

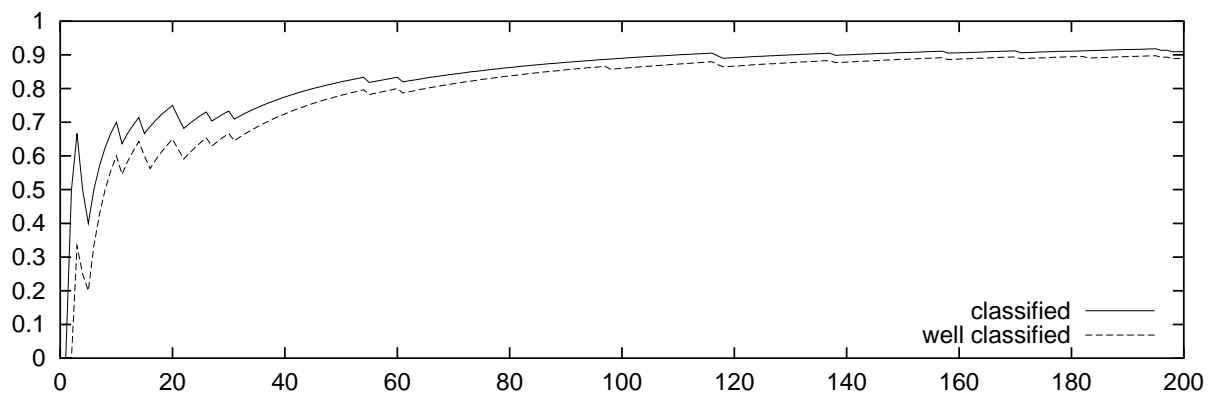

Figure 4. Filtering spams

The rates in Figure 4 are accumulated from the start of the experiment: $\operatorname{classified}(n)=\frac{\text { total classified }(n)}{n}$. So they take into account the bad rates of the transient phase. The pseudo-instantaneous rates, $\operatorname{classified}(n)=$ $\frac{\text { total classified }(n)-\text { total classified }(n-\delta t)}{\delta t}$ are over $90 \%$ after the $80^{\text {th }}$ message for a time window $(\delta t)$ of 50 messages (average number of messages per week during the experiment).

5.2.2.2 Classifying e-mail messages The second application is a variant of the first one in which keywords are not limited to two values. We classify email messages in about 20 non-exclusive categories such as teaching, research, spam, call-for-paper, and so on. Note that these categories were not fixed a priori, but appeared only when required by the meaning of incoming messages. Thus, the vocabulary of categories remains open for ever.

Figure 5 shows the results of this experiment. The "automatic" line shows the rate of automatic classification using rules that are suggested by the system and accepted by the user (40 rules, including 15 for spams). The "suggested" line shows the rate of correct suggested classification. It tends to decrease simply because the sum of the two rates must be less than 1 . Both rates are measured in number of features. The solid line shows the sum of the two rates. 


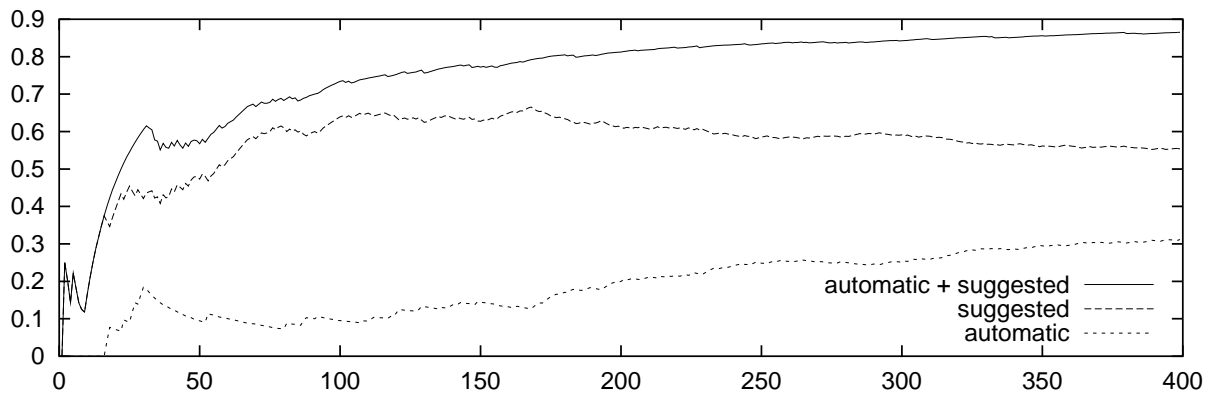

Figure 5. Classifying e-mail messages

Note again that these rates are cumulative; the instantaneous rates of "automatic+suggested" is constantly over $85 \%$ after the $100^{\text {th }}$ message for a time window of 50 messages.

\section{Implementation of a LIS}

Logic information systems are based on two choices: Concept Analysis and the use of an arbitrary logic. Both choices ask for performance questions as the complexity of concept lattices is exponential, and using logical descriptions implies the introduction of a theorem prover. However, theorem proving may be costly, e.g., exponential time for propositional logic.

\subsection{Concept analysis without concept lattice}

We first present the internal representation of a logical context we have chosen for our prototype; we also describe operations on this representation. Then, we give space complexity for the internal representation, and time complexity for the operations. These complexities are based on hypotheses, justified by experiments.

\subsubsection{Internal Representation of a Logical Context}

In a logical context $K=(\mathcal{O}, \mathcal{L}, d)$, we are mainly interested in navigation and querying. From their definition in Sections 3.3 and 3.4, it appears that all needed elementary operations are:

- $\sqsubseteq$ : subsumption test between two formulas,

- $\tau_{K}$ : extent of a formula, which is based on subsumption $\sqsubseteq$ and on the set of objects $\mathcal{O}$,

- $\cap$ : set intersection (between two extents), 
- $\operatorname{Max}_{\sqsubseteq}$ : selection of maximal formulas w.r.t. $\sqsubseteq$, from a set of formulas.

Three of these operations use the subsumption test, i.e., they call the theorem prover of the logic. As its complexity is high for some logics, it is preferable to limit its use as much as possible. In particular, the computation of extents is intensively used in the search for links, whereas each computation of an extent means as many subsumption tests as the number of objects.

We propose to store subsumption relations between features, and between features and object descriptions, in what we call a logical cache. We also propose to cache extents of features, which are intensively used for navigation. Thus, a logical context $K$ is stored as a directed acyclic graph whose nodes are logical formulas (features $F_{K}$ and object descriptions $d(\mathcal{O})$ ), equipped with their extent, and whose arcs are subsumption relations between these formulas (arcs deducible by reflexivity and transitivity are not represented). This representation is the Hasse diagram of the partially ordered set $\left\langle F_{K} \cup d(\mathcal{O})\right.$; $\sqsubseteq$.

Note that this representation is different from the concept lattice, which is usually used in systems based on concept analysis (e.g., Godin, Missaoui, and April, 1993; Cole and Stumme, 2000). We think the logical Hasse diagram is more appropriate to logical navigation for several reasons:

- the order used to compare a potential link to the working view and to select maximal links is the subsumption $\sqsubseteq$, and not the order on concepts $\leq^{c}$,

- it allows to search for navigation links among the features subsumed by the working view, without testing $x \sqsubset w v$; and similarly to select maximal features without actually checking subsumption,

- the number of concepts can be exponential with the number of objects, whereas the set of features is sufficient for navigation, and its size is linear with the number of objects (see Section 6.1.2),

- the order $\leq^{c}$ on concepts changes when objects are added, contrary to the order $\sqsubseteq$ on formulas.

The principal operations we consider on this logical cache are:

insertion of a formula: a new feature or object description is inserted in the graph and connected to other formulas according to the subsumption relation;

addition of an object: a new object is placed on the node representing its description, and extents of existing nodes are updated;

search for the maximal features satisfying some property: this is used to search for navigation links and views.

These operations are sufficient to implement the shell commands we presented for navigation and querying. 


\subsubsection{Theoretical and Practical Complexity}

The two important parameters for determining complexities are the number of objects, $n$, and the number of features per object, $f$ (there are other parameters, but they are bounded by $f$ ). The space complexity of the Hasse diagram is in $\mathcal{O}\left(f^{2} n\right)$. Among the three above operation, only the insertion of a formula actually uses the subsumption test: the number of call to $\sqsubseteq$ is in $\mathcal{O}(f n)$. Other operations find their results in the logical cache. The time complexity of set operations is either in $\mathcal{O}\left(f^{2}\right)$ (insertion of a formula, and addition of an object), or in $\mathcal{O}(f n)$ (search for links and views).

Our experiments have shown that the number of features per object $f$ is nearly constant for a given application (see Section 6.1.3). This is explained by the fact that features are extracted from object descriptions automatically and without considering other objects. The consequence for complexities is that every operation is either constant, or linear with the number of objects. This means that navigation and querying in a LIS are tractable.

\subsubsection{Experiments}

A prototype of a Logical Information System has been built for experimentation purpose. It has been implemented in $\lambda$ Prolog (Miller and Nadathur, 1986; Belleannee, Brisset, and Ridoux, 1999) as a generic system in which a theorem-prover and a syntax analyzer can be plugged-in for every logic used in descriptions. It is not meant to be efficient, though it can handle several thousand entries. Contrary to other tools based on concept analysis, it does not create the concept lattice. It only manages a Hasse diagram of the features used so far.

For the ICCS Bib context (see Example 7), the Hasse diagram has 954 nodes and 2150 arcs. In the experiments reported in this article, all response times are shorter than 1 second. In other experiments with a full-sized Bib context, i.e., all BibTeX fields (Lamport, 1985) are represented and there are several thousand bibliographical references, the Hasse diagram has an average of 15 nodes per object, 3 arcs per node, and a height of about 5 . This experiment and others support the idea that the number of features per object, $f$, is nearly constant for a given application; e.g., $f$ is about 60 in Bib contexts. This has a positive implication on the complexity of LIS operations, because under this hypothesis their time complexity is either constant, or linear with the number of objects (see above). 


\subsection{Logics for LCA and LIS}

We present in this section how the generic scheme that takes a logic as a parameter can be instantiated.

\subsubsection{Principles}

Using logics as schemas in data-bases implies that end-users or system administrators will have to define and implement logics. Clearly, this is out of reach for most of them. In order to make our principles practicable anyway, we have designed a framework for specifying and implementing logics. This framework is based on what we called logic functors. Using them, defining and implementing a logic consists merely in combining parameterized logics. Each logic functor consists in a logic component, e.g., propositional logic or interval comparison. Functors can be composed to form new logics, e.g., propositional logic on intervals.

Each functor is implemented as a parameterized theorem prover. Our goal is that the theory and theorem prover of a combination of functors result from a systematic combination of the theory and theorem prover of each functor.

All functors and their compositions implement a common interface which corresponds to the 6-tuple of Definition 1. This makes it possible to program generic applications that can be instantiated with a logic component. Conversely, customized logics built using the logic functors can be embedded in an application that respects this interface.

The whole framework development is geared towards manipulating logics as lattices. So, subsumption is considered as a relation between formulas, and we study the conditions under which this relation is a partial order.

Our idea is to consider that a logic interprets its formulas as functions of their atoms. By abstracting atomic formulas from the language of a logic we obtain what we call a logic functor. A logic functor can be applied to a logic to form a new logic. For instance, if propositional logic is abstracted over its atomic formulas, we obtain a logic functor called prop, which we can apply to, say, a logic on intervals interv, to form propositional logic on intervals, prop(interv). 


\subsubsection{Logics and logic functors}

We present the logic functor structure. More details can be found in (Ferré and Ridoux, 2002a), particularly on the conditions for composability.

Definition 43 (logic) $A$ logic $L$ is a triple $\left(A S_{L}, S_{L}, P_{L}\right)$ where $A S_{L}$ defines the abstract syntax of formulas of $L, S_{L}$ defines their semantics, and $P_{L}$ defines their interface.

$S_{L}$ is a pair $\left(I_{L}, \models_{L}\right)$ where

- $I_{L}$ is the interpretation domain of the formulas of $L$, and

- $={ }_{L} \in \mathcal{P}\left(I_{L} \times A S_{L}\right)$ is the satisfaction relation between interpretations and formulas; $i \models_{L} f$ means that $i$ is a model of $f$. We write $M_{L}(f)=\left\{i \in I_{L} \mid i \models_{L} f\right\}$ the set of all models of a formula $f$.

$P_{L}$ is a 5-uple $\left(\sqsubseteq_{L}, \sqcup_{L}, \sqcap_{L}, \top_{L}, \perp_{L}\right)$ where

- $\sqsubseteq_{L} \in \mathcal{P}\left(A S_{L} \times A S_{L}\right)$ is the subsumption relation,

- $\sqcup_{L}, \sqcap_{L} \in A S_{L} \times A S_{L} \rightarrow\left(A S_{L} \cup\{\right.$ undef $\left.\}\right)$ are conjunction and disjunction, and

- $\top_{L}, \perp_{L} \in A S_{L} \cup\{$ undef $\}$ are the tautology and contradiction for $L$.

All logics built with logic functors present the same interface $\left(\sqsubseteq_{L}, \sqcup_{L}, \sqcap_{L}, \top_{L}, \perp_{L}\right)$, plus other operations like updating (operation $\diamond$ in Section 4.1), parsing and printing. So, they implement the same abstract data-type. The 5 logic operations considered here correspond to a minimal requirement about logics; the ability to test subsumption, to build new formulas using conjunction and disjunction, and to determine if a formula is a tautology or a contradiction. A logic may have more connectives, but they will appear in its abstract syntax, not in its interface.

In order to simplify the presentation, we will only consider operations $\sqsubseteq_{L}, \sqcap_{L}$, and $\top_{L}$ in the sequel.

The definition of $\sqcap_{L}$ needs not be total. Similarly, $\top_{L}$ needs not be defined. Moreover, there is no a priori relation between $S_{L}$ and $P_{L}$. So, we need a notion of completeness and consistency that takes into account partial definitions.

Definition 44 (completeness) Let $L$ be a logic, the operations of its interface $P_{L}$, i.e., $\sqsubseteq_{L}, \sqcap_{L}$, and $\top_{L}$, are complete w.r.t. a semantics $S_{L}$, if and only if for all formulas $f, g \in A S_{L}$ we have

- $M_{L}(f) \subseteq M_{L}(g) \Longrightarrow f \sqsubseteq_{L} g$,

- $\top_{L} \neq$ undef $\Longrightarrow M_{L}\left(\top_{L}\right)=I_{L}$

- $f \sqcap_{L} g \neq$ undef $\left.\Longrightarrow M_{L}\left(f \sqcap_{L} g\right) \supseteq M_{L}(f) \cap M_{L}(g)\right)$, 
An interface $P_{L}$ is complete w.r.t. a semantics $S_{L}$ if and only if each of its operation is complete.

Definition 45 (consistency) Let $L$ be a logic, the operations of its interface $P_{L}$, i.e., $\sqsubseteq_{L}, \sqcap_{L}$, and $\top_{L}$, are consistent w.r.t. a semantics $S_{L}$, if and only if for all formulas $f, g \in A S_{L}$ we have

- $f \sqsubseteq L g \Longrightarrow M_{L}(f) \subseteq M_{L}(g)$,

- $T_{L}$ is always consistent,

- $f \sqcap_{L} g \neq$ undef $\Longrightarrow M_{L}\left(f \sqcap_{L} g\right) \subseteq M_{L}(f) \cap M_{L}(g)$,

An interface $P_{L}$ is consistent w.r.t. a semantics $S_{L}$ if and only if each of its operation is consistent.

By this definition, operations $\sqcap_{L}$ and $\top_{L}$ (and also $\sqcup_{L}$ and $\perp_{L}$ ) of a completely undefined interface are trivially complete and consistent, but they makes a useless interface. So, the game of designing a new logic is to make it defined enough to be useful, but still complete and consistent.

Definition 46 (logic functor) Assuming $\mathbb{L}, \mathbb{A} \mathbb{S}, \mathbb{S}$, and $\mathbb{P}$ the collections of all logics, abstract syntax, semantics, and interface, a logic functor $F: \mathbb{L}^{n} \rightarrow \mathbb{L}$ is a triple $\left(A S_{F}, S_{F}, P_{F}\right)$ defined as follows:

- $A S_{F}: \mathbb{A S}^{n} \rightarrow \mathbb{A} \mathbb{S}$ such that $A S_{F\left(L_{1}, \ldots, L_{n}\right)}=A S_{F}\left(A S_{L_{1}}, \ldots, A S_{L_{n}}\right)$,

- $S_{F}: \mathbb{S}^{n} \rightarrow \mathbb{S}$ such that $S_{F\left(L_{1}, \ldots, L_{n}\right)}=S_{F}\left(S_{L_{1}}, \ldots, S_{L_{n}}\right)$,

- $P_{F}: \mathbb{P}^{n} \rightarrow \mathbb{P}$ such that $P_{F\left(L_{1}, \ldots, L_{n}\right)}=P_{F}\left(P_{L_{1}}, \ldots, P_{L_{n}}\right)$,

By convention, a logic will be considered as a logic functor of type $\mathbb{L}$.

Logic functors are used as follows. $S_{L}$ describes the semantics; it acts as a specification. $P_{L}$ implements an interface; its description must be constructive enough, so that it leads directly to a program. A part of the interface describes how the concrete syntax is parsed and printed (remember that $A S_{L}$ is only the abstract syntax). The other part offers logic operations. The user composes a logic by applying logic functors to logics, say $F\left(L_{1}, \ldots, L_{n}\right)$, and a logic composer takes such an expression and produces automatically the concrete implementation of the logic by gluing together the concrete implementations of $F, L_{1}, \ldots$, and $L_{n}$. This results in a software component, with a formally specified interface, that can be plugged in any software system that assumes the same interface.

This methodology leads to designing a library of logic functors for describing objects of LIS: a unary propositional functor (prop), whose main quality is to make a total logic out of a partial one $(\operatorname{prop}($ partial $)=$ complete $)$, several nullary logic functors for concrete domains like strings and intervals, several n-ary logic functors for combining descriptions, and an auto-epistemic logic 
functor for representing (un)completeness of knowledge.

It is worth insisting on the auto-epistemic logic functor. Very often, users expect that a description author is "Smith" implies $\neg$ author is "Jones". However, this is false in standard propositional logic. One needs a form of Closed World Assumption or a kind of Negation as Failure to prove that. However, to be used in a LIS a logic must be monotonic (because its subsumption relation must form a lattice). $\mathcal{O N} \mathcal{L}$ (a.k.a. "All I Know" Levesque, 1990 ) is an epistemic modal logic that permits to express that a formula tells the whole truth. It is a variant of $\mathcal{O N} \mathcal{L}$ that we are using in LIS (Ferré, 2001); and its functor is called aik. Since it is very frequent to have to express absolute knowledge, the idiom $\operatorname{prop}(\operatorname{aik}(\operatorname{prop}(.))$.$) has become a mandatory$ prefix of the logics used in LIS.

The principle of composing logic functors has been implemented in a prototype. It reads logic specifications such as $\operatorname{prod}(\operatorname{prop}($ atom $), \operatorname{prop}($ interv)) and produces automatically a printer, a parser, and a theorem-prover. This example means that logic formulas are products of a proposition on atoms and a proposition on intervals. The prototype reads such a constructed logic and builds a theorem-prover for it by instantiating the theorem-prover associated to each logic functor at every occurrence where it is used. The prototype, each functor implementation, and the resulting implementations are written in $\lambda$ Prolog.

Coming back to the bibliography example of the introduction, we construct a dedicated logic with logic functors as follows:

$$
\operatorname{prop}\left(\operatorname{aik}\left(\operatorname{prop}\left(\operatorname{sum}\left(\text { atom, valattr }\left(\operatorname{sum}_{n}(\text { interv, } \operatorname{string}, \ldots)\right)\right)\right)\right)\right) \text {. }
$$

which means propositional logic on epistemic modal formulas on propositional logic on a sum (i.e., a mix) of atomic formulas and valued attributes whose values are themselves made of interval formulas, string formulas, etc. According to the theory of logic functors, the following theorem holds.

Theorem 47 (Bib logic is ok) The logic L used in Example \%,

$L=\operatorname{prop}\left(\operatorname{aik}\left(\operatorname{prop}\left(\operatorname{sum}\left(\operatorname{atom}, \operatorname{valattr}\left(\operatorname{sum}_{2}(\right.\right.\right.\right.\right.$ interv, string $\left.\left.\left.\left.\left.)\right)\right)\right)\right)\right)$,

is such that $P_{L}$ is total, bounded, and consistent and complete in all five operations w.r.t. $S_{L}$. 


\section{Conclusion}

\subsection{Summary of LIS}

We have presented the specifications of a Logical Information System based on (Logical) Concept Analysis. It is based on Concept Analysis and is generic w.r.t. a logic for describing objects. In this framework, navigation/querying and creation/updating can be seamlessly integrated.

In this way, standard commands of a file system shell can be mimicked in a logical context. However, a simple generalization of the definition of links forms a framework in which operations of data-analysis or data-mining can also be expressed. Using this framework, purely symbolic navigation as well as statistical exploration can be integrated smoothly as variants of the same generic operation.

As opposed to previous attempts of using Concept Analysis for organizing data, we do not propose to navigate directly in the concept lattice. Instead, we use the contextualized logic (i.e., the logical view of the concept lattice) to evaluate the relevance of navigation links. Those that do not narrow the focus of the search are called views. They only restrict the language of available navigation links. Other links, that do narrow the focus of the search, can be used to come closer to some place of interest. The definition of links can be generalized to encompass data-mining notions like necessary and sufficient conditions, and association rules.

The advantage of LIS is a great flexibility which comes from two factors:

(1) the integration of operations that were exclusive in most systems,

(2) the use of logic with Concept Analysis, which solves the name problem.

We have experimented it in various contexts: e.g., cook-books, bibliographical repository, software repository (search by keywords, and search by types), and simply a note-pad. Various logic components were used in these contexts: atoms, intervals, strings, propositional logic, type entailment, taxonomies (e.g., for ingredients). In all cases, a LIS goes beyond any a priori structure and permits many kinds of views on the same information. For instance, in the case of a cook-books, if every recipe is described by its ingredients, its process, the required kitchen utensils, its dietetic value, its place in a meal, and more cultural information, then a cook, a dietician, and a gourmet can have very different views on the same data, and acquire new information by data-mining and learning, simply by using a few LIS shell commands. Similarly, if software components have intrinsic descriptions like their types and languages, the modules they use, parts of specification, and requirements, and 
extrinsic descriptions like their testing status, and who is using them, then several software engineering operations like developing and testing, versioning and configuring, and maintenance and evolution can be done using the same repository under different views, and also going smoothly from one view to another.

A thorough description of LIS can be found in the $\mathrm{PhD}$ thesis of the first author (Ferré, 2002b). It contains all proofs, gives more details about implementation, and in addition to this paper, considers the representation of complete vs. incomplete object descriptions.

The exclusive use of logic for describing objects, and for designing operations asks the question of whether uncertainty can be dealt with in LIS. In fact, LIS can handle uncertainty at two levels.

First level is the logical language. Because LIS is generic w.r.t. logic, it can accomodate uncertainty either in descriptions of objects or in queries by using logics that handle uncertainty. Even propositional logic can handle a kind of uncertainty with disjunctions, but other logics like interval logic or logic "All I Know" can handle other kinds of uncertainty. Intervals can deal with uncertainty of numerical values, and "All I Know" deals with the extent of knowledge. Both have been implemented as logic functors (see Section 6.2).

LIS is not opposed to using fuzzy modeling languages (logic, sets, etc), but it requires an entailment relation that forms a lattice. In particular, the entailment relation must be monotonic. Note that "All I Know" is a monotonic logic that handles a notoriously non-monotonic feature: the "Closed World Assumption". We take this as a hint that the restriction to monotonic logics is not a restriction in expressiveness.

Second level is the data-analysis level. As we have shown in Section 5.1, it is very natural to generalize navigation into data-analysis w.r.t. to indicators like confidence and support. This makes it possible to extract knowledge that is not necessarily $100 \%$ true.

\subsection{Related Works}

There have been several other proposal of navigation/querying based on Concept Analysis. Lindig (1995) designed a concept-based component retrieval based on sets of significant keywords which are equivalent to our links for the logic of attributes underlying FCA. Godin et al. (1993) propose a direct navigation in the lattice of concepts, which is in fact very similar to Lindig's approach except that only greatest significant keywords, according to the contextualized subsumption on attributes, are displayed to the user. They have 
also notions common to our LIS such as working query, direct query specification, and history of selected queries.

Cole and Stumme (2000) developed a Conceptual E-mail Manager (CEM) where the navigation is based on Conceptual Scales (Prediger, 1997; Prediger and Stumme, 1999). These scales are similar to our views in the sense that they select some attributes acting as links and displayed, as for us, with the size of the concept they select. A difference with our LIS is that these links are ordered according to concept lattices of scales, but it can also be done in LIS by a post-treatment on answers of command 1 s.

However, the main difference with all of these approaches is that we use an (almost) arbitrary logic to express properties. This enables us to have automatic subsumption relations (e.g., (author is "Wille, Mineau") $\sqsubseteq$ (author contains "Wille") $\sqsubseteq$ (author)), and thus some implicit views (e.g., author, year). Another difference is that we propose to handle in a uniform way, based on $\mathrm{CA}$, navigation and querying as above, but also, updating, data-mining, learning, etc.

van Rijsbergen (1986) also combined information retrieval (IR) and logic by introducing a logical model of IR, in which both object descriptions and queries are logical. As for us, many logics could be used in this model, but a first difference is that the relevance relation is defined as a measure combining the exhaustivity and specificity of the object description to the query, rather than an exact one (the subsumption $\sqsubseteq$ ). This measure is used to rank the answers of a query. A second difference is that navigation is not here combined with querying. Later, Chiaramella (1997) added some navigation to this logical model of IR. However, this navigation is structural rather than conceptual as in a LIS, which means that a navigation step leads from a set of objects to another, rather than from a query to a set of sub-queries. The fact that our answers are intentional rather than extensional justifies we can rely on an exact relevance relation because answers to queries are not flat sets of objects. However, it is also possible to take into account uncertainties in a LIS (see the end of Section 7.1).

\subsection{Future Work}

Our most practical perspective is to design a logical file system, which would implement the ideas we have presented in this article, and serve as a Logic Concept Repository for a LIS. The expected advantage is to offer the services described here at a standard system level that is accessible for every application. So doing, even applications that do not know about logical information systems (like e.g., all existing compilers) would benefit from it. For this logi- 
cal file system, it will also be important to make our ideas work with a large volume of data in an efficient way.

A graphical user-interface to logical file systems would allow to display in an integrated fashion the working query, the working view, and the corresponding extent and set of links. For instance, a graphical interface for keeping trace of navigation, like what is becoming standard for file browsers, has been already experimented for a simple logic (attributes with values) but should be developed further. This amounts to keep a trace of the path from the start of the navigation to the current place. Moreover, the set of links could be presented graphically as a diagram of ordered formulas. A further refinement is to take into account the contextualized subsumption, to get something similar to concept lattices derived from scales (Cole and Stumme, 2000). This amounts to represent an overview of possible future navigations.

The World Wide Web can also be explored using our techniques if one considers answers to web-queries as a formal context into which to navigate. More ambitious is to think of a Web-based LIS. In this case, the main issues will be distribution of data and computation.

An application of the learning schema exposed in Section 5.2.1 is to use the learning schema for navigating. A description would play the role of a query, and its associative concepts could be proposed to the user as alternative queries. The advantage is that though the initial query could have an empty answer, the alternative ones always correspond to non-empty concepts. So, it makes it possible to start a search with only an example of what the user is looking for, and then see actual representatives of the queried concepts.

Another interesting perspective follows the observation that the notion of associative concepts is closely related to modified and new concepts in the incremental concept formation (Godin, Missaoui, and Alaoui, 1995). We developed further this correspondence, which led to an improved algorithm for incrementally computing concepts of sparse contexts (Ferré, 2002a).

In all this article, names or descriptions are essentially unary predicates, whichever is the actual logic used for this purpose. However, several applications require to express relations between objects, i.e., n-ary predicates. For instance, a LIS for a software environment should permit to express such relations as calls $f$ or is connected to $x$, where $f$ and $x$ are objects. These relations form concrete links between objects, which we plan to consider for navigation in a future work. The main difficulty is to manage the concrete links in a way that remains compatible with the other navigation links. This will also permit to represent topological informations, e.g., West of $x$ or 10 miles from $y$, that are used in Geographical Information Systems. 


\section{References}

Barbut, M., Monjardet, B., 1970. Ordre et classification - Algèbre et combinatoire (2 tomes). Hachette, Paris.

Belleannee, C., Brisset, P., Ridoux, O., 1999. A pragmatic reconstruction of $\lambda$ prolog. The Journal of Logic Programming 41, 67-102.

Brachman, R. J., 1979. On the epistemological status of semantic nets. In: Findler, N. V. (Ed.), Associative Networks: Representation of Knowledge and Use of Knowledge by Examples. Academic Press, New York.

Chaudron, L., Maille, N., 1998. 1st order logic formal concept analysis: from logic programming to theory. Computer and Information Science 13 (3).

Chiaramella, Y., 1997. Browsing and querying: two complementary approaches for multimedia information retrieval. In: N. Fuhr, G. Dittrich, K. T. (Ed.), Hypermedia - Information Retrieval - Multimedia. Universitätsverlag Konstanz, pp. 9-26.

Cole, R., Stumme, G., 2000. CEM - a conceptual email manager. In: Mineau, G., Ganter, B. (Eds.), Int. Conf. Conceptual Structures. LNCS 1867. Springer, pp. 438-452.

Crestani, F., Lalmas, M., 2001. Logic and uncertainty in information retrieval. In: Lectures in Information Retrieval. LNCS 1980. pp. 179-207.

Davey, B. A., Priestley, H. A., 1990. Introduction to Lattices and Order. Cambridge University Press.

Di Cosmo, R., 1995. Isomorphisms of Types: from $\lambda$-calculus to information retrieval and language design. Progress in theoretical computer science. Birkhäuser.

Ferré, S., 2001. Complete and incomplete knowledge in logical information systems. In: Benferhat, S., Besnard, P. (Eds.), Symbolic and Quantitative Approaches to Reasoning with Uncertainty. LNCS 2143. Springer, pp. 782791.

Ferré, S., Oct. 2002a. Incremental concept formation made more efficient by the use of associative concepts. Research Report RR-4569, Inria, Institut National de Recherche en Informatique et en Automatique.

URL http://www.inria.fr/RRRT/RR-4569.html

Ferré, S., Oct. 2002b. Systèmes d'information logiques : un paradigme logico-contextuel pour interroger, naviguer et apprendre. Thèse d'université, Université de Rennes 1, accessible en ligne à l'adresse http://www.irisa.fr/bibli/publi/theses/theses02.html.

URL http://www.irisa.fr/bibli/publi/theses/theses02.html

Ferré, S., Ridoux, O., Dec. 1999. Une généralisation logique de l'analyse de concepts formels. Technical Report RR-3820, Inria, Institut National de Recherche en Informatique et en Automatique, an english version is available at http://www.irisa.fr/lande/ferre.

Ferré, S., Ridoux, O., 2000. A logical generalization of formal concept analysis. In: Mineau, G., Ganter, B. (Eds.), Int. Conf. Conceptual Structures. LNCS 1867. Springer, pp. 371-384. 
Ferré, S., Ridoux, O., 2002a. A framework for developing embeddable customized logics. In: Pettorossi, A. (Ed.), Int. Work. Logic-based Program Synthesis and Transformation. LNCS 2372. Springer, pp. 191-215.

Ferré, S., Ridoux, O., 2002b. The use of associative concepts in the incremental building of a logical context. In: U. Priss, D. Corbett, G. A. (Ed.), Int. Conf. Conceptual Structures. LNCS 2393. Springer, pp. 299-313.

Ganter, B., Kuznetsov, S., 2001. Pattern structures and their projections. In: Delugach, H. S., Stumme, G. (Eds.), Int. Conf. Conceptual Structures. LNCS 2120. Springer, pp. 129-142.

Ganter, B., Wille, R., 1999. Formal Concept Analysis — Mathematical Foundations. Springer.

Gifford, D. K., Jouvelot, P., Sheldon, M. A., O’Toole, J. W. J., 1991. Semantic file systems. In: 13th ACM Symposium on Operating Systems Principles. ACM SIGOPS, pp. 16-25.

Godin, R., Missaoui, R., Alaoui, H., 1995. Incremental concept formation algorithms based on Galois (concept) lattices. Computational Intelligence 11 (2), 246-267.

Godin, R., Missaoui, R., April, A., 1993. Experimental comparison of navigation in a Galois lattice with conventional information retrieval methods. International Journal of Man-Machine Studies 38 (5), 747-767.

Gopal, B., Manber, U., 1999. Integrating content-based access mechanisms with hierarchical file systems. In: third symposium on Operating Systems Design and Implementation. USENIX Association, pp. 265-278.

Herzig, A., Rifi, O., 1999. Propositional belief update and minimal change. Artificial Intelligence 115 (1), 107-138.

Keller, A. M., Mar. 1985. Algorithms for translating view updates to database updates for views involving selections, projections, and joins. In: 4th ACM Symp. Principles of Database Systems. pp. 154-163.

Krone, M., Snelting, G., May 1994. On the inference of configuration structures from source code. In: Int. Conf. Software Engineering. IEEE Computer Society Press, pp. 49-58.

Kuznetsov, S., 1999. Learning of simple conceptual graphs from positive and negative examples. In: Żytkow, J. M., Rauch, J. (Eds.), Principles of Data Mining and Knowledge Discovery. LNAI 1704. Springer, pp. 384-391.

Kuznetsov, S., Objedkov, S., 2001. Comparing performance of algorithms for generating concept lattice. In: et al., E. M. N. (Ed.), ICCS-2001 Int. Workshop on Concept Lattices-based Theory, Methods and Tools for Knowledge Discovery in Databases. Stanford University, CRIL - IUT de Lens, France.

Lamport, L., 1985. LATEX - A Document Preparation System. AddisonWesley, 2nd edition.

Levesque, H., Mar. 1990. All I know: a study in autoepistemic logic. Artificial Intelligence $42(2)$.

Lindig, C., 1995. Concept-based component retrieval. In: IJCAI95 Workshop on Formal Approaches to the Reuse of Plans, Proofs, and Programs.

Meghini, M., Sebastiani, F., Straccia, U., Thanos, C., 1993. A Model of infor- 
mation Retrieval based on Terminological Logic. In: 16th Annual Int. ACM SIGIR Conference on Research and Development in Information Retrieval. pp. 298-307.

Miller, D. A., Nadathur, G., 1986. Higher-order logic programming. In: Shapiro, E. (Ed.), In Third Int. Conf. Logic Programming. LNCS. SpringerVerlag, London, pp. 448-462.

Napoli, A., Dec. 1997. Une introduction aux logiques de descriptions. Rapport de recherche RR-3314, Inria, Institut National de Recherche en Informatique et en Automatique.

Prediger, S., 1997. Logical scaling in formal concept analysis. LNCS 1257 , 332-341.

Prediger, S., Stumme, G., 1999. Theory-driven logical scaling. In: International Workshop on Description Logics. Vol. 22. Sweden.

Snelting, G., Jul. 1998. Concept analysis - A new framework for program understanding. ACM SIGPLAN Notices 33 (7), 1-10.

Ullman, J. D., 1989. Principles of Database and Knowledge-Base Bystems. Computer Science Press, Rockville, Maryland.

van Rijsbergen, C. J., 1986. A new theoretical framework for information retrieval. In: Int. ACM SIGIR Conference on Research and Development in Information Retrieval. ACM, pp. 194-200.

Vogt, F., Wille, R., 1994. TOSCANA - a graphical tool for analyzing and exploring data. In: Symposium on Graph Drawing. LNCS 894. pp. 226-233.

Wille, R., 1982. Ordered Sets. Reidel, Dordrecht Boston, Ch. Restructuring lattice theory: an approach based on hierarchies of concepts, pp. 445-470. 